NBER WORKING PAPER SERIES

\title{
COMPLEMENTS VERSUS SUBSTITUTES AND TRENDS IN FERTILITY CHOICE IN DYNASTIC MODELS
}

\author{
Larry E. Jones \\ Alice Schoonbroodt \\ Working Paper 13680 \\ http://www.nber.org/papers/w13680
}

\author{
NATIONAL BUREAU OF ECONOMIC RESEARCH \\ 1050 Massachusetts Avenue \\ Cambridge, MA 02138
}

December 2007

We thank the National Science Foundation for financial support and Martin Gervais, John Knowles, Jeremy Lise, and Michèle Tertilt as well as seminar participants at SITE 2007, Vienna Macro Workshop 2007, NYU and CEMFI for their comments. We also thank Michael Bar and Oksana Leukhina for their valuable comments and for making their data available to us and Anderson Schneider for valuable research assistance. The views expressed herein are those of the author(s) and do not necessarily reflect the views of the National Bureau of Economic Research.

(C) 2007 by Larry E. Jones and Alice Schoonbroodt. All rights reserved. Short sections of text, not to exceed two paragraphs, may be quoted without explicit permission provided that full credit, including (C) notice, is given to the source. 
Complements versus Substitutes and Trends in Fertility Choice in Dynastic Models

Larry E. Jones and Alice Schoonbroodt

NBER Working Paper No. 13680

December 2007

JEL No. E13,J11,J13,O11

\begin{abstract}
$\underline{\text { ABSTRACT }}$
The Barro-Becker model is a simple intuitive model of fertility choice. In its original formulation, however, it has not been very successful at reproducing the changes in fertility choice in response to decreased mortality and increased income growth that demographers have emphasized in explaining the demographic transition. In this paper we show that this is due to an implicit assumption that number and utility of children are complements, which is a byproduct of the high intertemporal elasticity of substitution (IES) typically assumed in the fertility literature. We show that, not only is this assumption not necessary, but both the qualitative and quantitative properties of the model in terms of fertility choice change dramatically when substitutability and high curvature are assumed. To do so, we first derive analytical comparative statics and perform quantitative experiments. We find that if IES is less than one, model predictions of changes in fertility amount to about two-thirds of those observed in U.S. data since 1800. There are two major sources to these predicted changes, the increase in the growth rate of productivity which accounts for about 90 percent of the predicted fall in fertility before 1880, and changes in mortality which account for 90 percent of the predicted change from 1880 to 1950 .
\end{abstract}

Larry E. Jones

Department of Economics

University of Minnesota

1035 Heller Hall

Minneapolis, MN 55455

and NBER

lej@econ.umn.edu

Alice Schoonbroodt

University of Southampton

United Kingdom

alicesch@soton.ac.uk 


\section{Introduction}

The seminal papers by Barro and Becker (Becker and Barro (1988) and Barro and Becker (1989)) have generated increasing interest in the study of the macroeconomics of fertility choice. The model is intuitive and analytically tractable. In its original formulation, however, it has not been very successful in generating the desired effects of changes in mortality and productivity on fertility choices and population growth rates (see Doepke (2005), Bar and Leukhina (2005)) as emphasized by demographers. This original formulation has also been adapted to include a variety of other interesting effects, such as the introduction of an educational choice and the related quantity-quality trade-off or the idea that children are partially an investment.

In this paper we show that one of the key parameters in dynastic models with Barro-Becker type altruism is the intertemporal elasticity of substitution (IES). We develop a simple model of fertility choice based on Barro-Becker type altruism without capital. While the original Barro-Becker formulation assumes very high levels of IES, we allow for the full range of parameter values. We then derive analytical and quantitative results that show why and how changes in infant, youth and adult mortality as well as productivity growth rates produce the kinds of effects suggested by demographers when low values of IES (closer to the growth and real business cycle literatures) are considered.

There are two main reasons why the choice of the IES matters for qualitative as well as quantitative results. The first effect comes from implicit complementarity/substitutability assumptions in these preference formulations. High IES, as in the original Barro-Becker formulation, implicitly introduces the assumption that family size and utility of children are complements in utility. To the contrary, low IES implies that they are substitutes in utility. This has important implications for the sign of first order effects. For instance, if number and utility of children are complements, the marginal utility of an extra child increases when productivity growth (and hence utility of children) increases. All else equal, this implies an increase in fertility and the population growth rate. With low elasticity and substitutability, the opposite is true.

The second effect is related to children being partly an investment good in these models and is most important for the size of the fertility response to changes in mortality and productivity. Here, much of what we find comes from basic intuitions about the desirability of changes in the growth rate of consumption for different values of IES - again, when IES is low, the value of increasing the growth rate of consumption is also low. In a dynastic model the principal effect of permanently increasing fertility, that is, the population growth rate, is to permanently increase growth rates in dynasty aggregates. Hence, because of the low IES any change in economic environment that facilitates this growth is met with a higher fraction of output going to current consumption and less to the investment good - fertility. This causes the stock of population to grow more slowly than with high values for the IES. 
We first revisit the properties of Barro-Becker preferences when all preference configurations are allowed. Along the way, we derive simple comparative statics at the household level to highlight the effect of the complementarity versus substitutability assumption for changes that exogenously increase children's utility. We then introduce longevity through random survival of adults ${ }^{1}$ as well as child mortality to derive the dynasty's utility and law of motion for population. ${ }^{2}$ This allows us to show how the choice of high elasticity leads to number and utility of children being complements and vice versa.

Next we solve the resulting dynastic planner's problem with exogenous productivity growth and derive comparative statics across balanced growth paths. Here we reinterpret increases in young age survival as decreases in the cost of surviving children. This allows us to compare qualitative predictions of the model that differ between population growth rates, surviving children and total births. What we find is that in the dynastic model both the qualitative and quantitative properties of fertility choice are highly sensitive to the choice of the IES and hence complementarity versus substitutability. In particular, we find:.

1. The effect of an increase in the growth rate of productivity is to increase population growth and fertility if the IES is greater than one, give no change if the IES equals one and decrease both population growth rates and surviving fertility if the IES is less than one.

2. A reduction in youth mortality decreases the cost of producing a surviving child and hence, increases surviving fertility and population growth rates for any value of IES. However, the size of this increase is increasing in the IES in a quantitatively significant way. This also implies that the decrease in total births tends to be larger for low IES.

3. Finally, we study the effects on fertility of increased life length. We find that increasing expected work life conditional on reaching adulthood increases population growth rates, but decreases surviving fertility. Again, the increase in population growth rates is increasing in the IES, while the decrease in surviving births is decreasing in the IES.

Since population growth rates slightly decreased over the last 200 years, while the number of births decreased substantially, these comparative statics results are interesting, both in a qualitative and in a quantitative sense. Qualitatively, commonly used values for the IES in the growth and RBC literatures also produce the observed direction of changes in fertility choice in response to changes in productivity growth. Quantitatively, "counterfactual" changes in population growth rates in response to changes in mortality are small when the IES is low - a puzzle often encountered in the fertility literature.

\footnotetext{
${ }^{1}$ In order to keep the model simple we follow Blanchard (1985) in making death a random occurrence but assume that all alive agents are identical. This allows us to omit the age distribution as a state variable and greatly simplifies the analysis.

${ }^{2}$ This is similar to Alvarez (1999) but with the addition of youth mortality and longevity.
} 
Next, we study quantitative comparative statics of these changes on fertility and population growth rates. First we calibrate child costs in the model to match the recent fertility experience in developed countries given their economic circumstances. That is, fertility producing roughly zero population growth in an environment where productivity growth is 2 percent per year, infant and child mortality rates are close to zero and expected working lifetime is about 45 years. Here, again, we find that the values of the costs of raising children are very sensitive to the choice of the IES: costs of children required to match any given population growth rate are sharply decreasing in the IES. This has been a problem in the fertility literature commonly using high values of IES.

We then perform several experiments using changes in economic environment that roughly correspond to changes over the past 200 years in developed countries. For high values of IES (of the order of 2), both the population growth rate and the Crude Birth Rate (CBR) increase substantially, contrary to the historical experience. For intermediate values of IES (of the order of 1) changes in productivity growth have no effect while changes in survival to adulthood decrease fertility. However since this decrease is not large, the model predicts that population growth rates increase substantially. Versions of the model with low IES (of the order of one third) have interesting and realistic quantitative implications in line with the analytical comparative statics above.

Finally, we simulate the U.S. experience since 1800, taking the timing of events and all three changes in economic environment into account. We find that overall, the model predicts that CBR would fall from 36.3 to 17.2 births per 1000 population. This corresponds to about two-thirds of the observed change in the U.S.. In terms of population growth rates, the model predicts a fall from 1.4 to 0.65 percent per year which captures about one half of observed changes in the U.S.. Interestingly, about 90 percent of model predicted changes in fertility before 1880 are accounted for by changes in productivity growth rates, while changes in mortality account for about 90 percent of the predicted fertility decrease thereafter. This finding speaks to the debate about what the root cause of the fertility decline was - was it reductions in youth mortality rates or was it accelerating economic development? What we find is: it was a combination of both with the latter being the most important factor early in the transition and mortality being the most important factor later on - a key assumption being low intertemporal elasticity of substitution in consumption.

The remainder of the paper is organized as follows. Section 2 reviews the literature. Section 3 revisits parameter restrictions in preferences and gives intuitive results relating to substitutability and complementarity. In Section 4 we setup and solve the dynastic planner's problem, impose balanced growth and derive analytical comparative statics for population growth, surviving children and all births. Section 5 presents quantitative comparative statics and Section 6 presents model predictions for the U.S. experience since 1800. In Section 7 we discuss various extensions. Section 8 concludes. 


\section{Related Literature}

Barro and Becker's original formulation has also been adapted to include a variety of other interesting effects, such as the introduction of an educational choice and the related quantity-quality trade-off or the idea that children are partially an investment. Examples of the educational choice literature include Becker, Murphy and Tamura (1990), Benhabib and Nishimura (1993), Galor and Weil (2000), Fernandez-Villaverde (2001), Soares (2003), Doepke (2004), Manuelli and Seshadri (2006), Rosenzweig (1990) and examples of children as an investment good are Ehrlich and Lui (1991), Boldrin and Jones (2002), Boldrin, DeNardi and Jones (2005). We address quantitative studies below. Most of these follow Barro-Becker utility parameter restrictions. Hence, results in this paper shed interesting new light on these extensions.

More recently several authors have studied quantitative versions of the BarroBecker model to examine its ability to track the basic trends over the last 200 years in fertility choices. These include Mateos-Planas (2002), Doepke (2005), and Bar and Leukhina (2005). They study the quantitative response of fertility in the model to changes in environment like infant, youth and adult mortality and changing productivity growth rates in a variety of different, but related economic environments. Surprisingly, they reach very different conclusions. For example:

1. In Mateos-Planas (2002) changes in mortality have only small effects on population growth and hence, he concludes that changes in productivity growth and/or changes in the cost of child-rearing are more likely to be the sources of the changes in fertility seen in the data.

2. Doepke (2005) also finds that reductions in child mortality appear not to be responsible for the observed changes in fertility. In fact, the predicted change in births is small and the model also has the counterfactual implication that population growth rates and surviving fertility increase substantially.

3. Bar and Leukhina (2005) study both the effects of changes in young-age mortality and productivity growth and conclude that the observed decreases in mortality are responsible for a large fraction of the reduction in fertility, but that changes in rates of productivity growth have very little effect.

The key difference in the assumptions that these authors make is about the size of the intertemporal elasticity of substitution in consumption (IES below). MateosPlanas assumes that this is less than unity but focuses only on population growth rates rather than births, Doepke assumes that the IES is greater than unity and Bar and Leukhina use a value equal to one. In this paper we develop a simple model of fertility choice based on Barro-Becker type altruism without capital but allowing for the full range of preference parameter values. This simplification allows us to derive analytical and quantitative results that help to reconcile the findings of these authors.

Other papers have also studied the quantitative properties of fertility models but have focused on different channels and setups. In Fernandez-Villaverde (2001), the effects of the falling relative price of capital and infant mortality rates are studied in 
an environment in which skill type is endogenous and skilled labor and capital are complements. In Doepke (2004) the impact of child labor and compulsory schooling laws are studied. In De la Croix and Doepke (2003) the role of income inequality is examined. Greenwood and Seshadri (2002) study the impact of changes in productivity in agriculture and manufacturing in a model where food is a necessity. Eckstein, Mira and Wolpin (1999) study the effects of changes in child mortality but in a lifecycle model without Barro-Becker altruism. Kalemli-Ozcan (2002, 2003) analyzes the effect of decreased child mortality in a stochastic fertility model focusing on the decreased risk that follows when a binomial probability approaches 1 . She concludes that changes in survival probabilities significantly decreases fertility. Doepke (2005), however, shows that this effect is greatly diminished when sequential fertility choices are allowed (see also Sah (1991), Wolpin (1999) and Ben-Porath (1976)).

\section{Barro-Becker altruism revisited}

In this section, we analyze Barro-Becker type preferences and their properties related to the intertemporal elasticity of substitution. First we show that one implicit restriction, namely positive utility, has lead to the assumption of complementarity between number and utility of children and in turn high intertemporal elasticity of substitution (IES) in the fertility literature. This restriction can be relaxed to also allow substitutability between number and utility of children for low values of IES. This assumption is crucial in determining the fertility response to permanent changes in productivity growth, youth mortality and longevity.

\subsection{The household problem: complements versus substitutes}

The standard presentation of the Barro-Becker model usually begins with a description of the preferences of a period $t$ adult. It is assumed that parents care about three separate objects:

i) their own consumption in the period, $c_{t}$,

ii) the number of children they have, $n_{t}$, and,

iii) the average utility of their children, $U_{t+1}$.

This is usually specialized further. It is assumed that utility of the typical time $t$ household is of the form:

$$
U_{t}=u\left(c_{t}\right)+\beta g\left(n_{t}\right) \sum_{i=1}^{n_{t}} \frac{1}{n_{t}} U_{i t+1}
$$

where $U_{i t+1}$ is the utility of the i-th child of the parent. Assuming that $U_{i t+1}=$ $U_{i^{\prime} t+1}=U_{t+1}$ for all $i, i^{\prime}$ this simplifies to:

$$
U_{t}=u\left(c_{t}\right)+\beta g\left(n_{t}\right) U_{t+1} .
$$


Intuitively, it makes sense to assume:

1.) Parents like the consumption good:

utility is increasing and concave in own consumption;

2.) Parents are altruistic:

holding $n_{t}$ fixed and increasing $U_{t+1}$ increases (strictly) the utility of the parent;

3.) Parents like having children:

holding $U_{t+1}$ fixed and increasing $n_{t}$ increases (strictly) the utility of the parent.

4.) The increase described in 3.) is subject to diminishing returns.

The first requirement is straightforward. It is satisfied as long as $u$ is increasing and concave. The second has implications for what $g$ can be. Since $\partial(u(c)+$ $\beta g(n) U) / \partial U=\beta g(n)$, it follows that (2.) implies that $g(n)>0$ for all $n$. The third requirement is less straightforward. Although this requirement makes intuitive sense, there are, because of the special restrictions implicit on functional forms, some issues that arise. For example, suppose that $U_{t+1}>0$. Then (3.) implies that $g(n)$ must be increasing in $n$. On the other hand, if $U_{t+1}<0$, (3.) implies that $g(n)$ should be decreasing in $n$. It follows that if it is possible for $U_{t+1}$ to be either positive OR negative, it is impossible to satisfy all of these requirements simultaneously. In sum, (1.)-(3.) are mutually inconsistent without some sort of restrictions on the possible values for $U_{t+1}$. Similar issues arise with respect to (4.). If $U_{t+1}$ is restricted to be positive, (4.) requires $g$ to be concave while if $U_{t+1}$ is restricted to be negative, (4.) requires that $g$ is convex.

This is not to say that these conditions cannot be satisfied. We must simply assume that either $U_{t+1}>0$ always or $U_{t+1}<0$ always and then make the appropriate assumptions on $g$. Without an assumption like this, the natural monotonicity properties of utility cannot be guaranteed. Thus, we are left with two options:

I. Assume $g(n)$ is non-negative, strictly increasing and concave and $U>0$, or

II. Assume $g(n)$ is non-negative, strictly decreasing and convex and $U<0$.

As it turns out, the choice between these two options has important implications for the properties of the model. This can be illustrated in a simple example.

First, consider how the solution of the problem of a time zero parent changes when the growth rate in wages is changed. In the simplest case, they face a problem of the form:

$$
\begin{aligned}
\max _{\left\{c_{0}, n_{0}\right\}} & u\left(c_{0}\right)+\beta g\left(n_{0}\right) U_{1} \\
\text { s.t. } & c_{0}+\theta_{0} n_{0} \leq w_{0},
\end{aligned}
$$

where $\theta_{0}$ is the cost of raising a child to survive to adulthood and $w_{0}$ is the wage rate in time 0 .

Increased wage growth only enters this problem through the indirect effect of changing $U_{1}$. That is, if wages grow faster, future generations will have larger choice sets and hence, $U_{1}$ will be larger. The first order condition for this problem is: 
Figure 1: Comparative Statics with respect to $U_{t+1}$

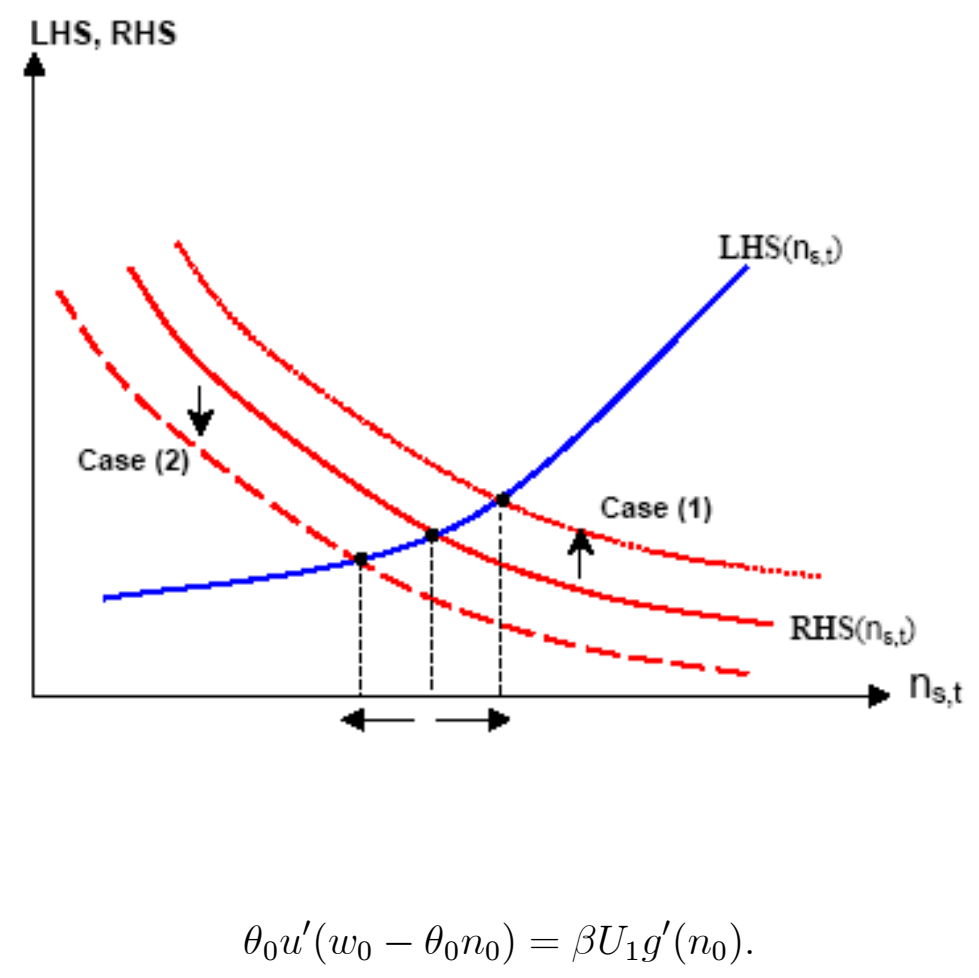

The left hand side of this equation is the marginal cost in terms of period 0 consumption of having an extra child and is increasing in $n_{0}$, while the right hand side is the marginal benefit and is decreasing.

A change in $U_{1}$ has different effects depending on which case we are in. In particular, whether an increase in $U_{1}$ increases or decreases the right hand side depends on whether $U_{1}$ is positive or negative. That is:

$$
\frac{\partial R H S\left(n_{0}, U_{1}\right)}{\partial n_{0} \partial U_{1}}=\beta g^{\prime}\left(n_{0}\right)=\frac{\partial^{2} U_{0}}{\partial n_{0} \partial U_{1}} .
$$

When $U_{1}$ is positive, $g$ is increasing and hence, the cross partial in 1 is positive - children and the utility of children are complements in the utility of the parent. ${ }^{3}$ In this case, it follows that a change in wage growth shifts the right hand side up causing $n_{0}$ to increase. Fertility is increasing in the rate of growth of wages. When $U_{1}$ is negative, $g$ is decreasing and hence, this is negative - children and the utility of children are substitutes in utility. In this case, it follows that the right hand side shifts down and $n_{0}$ falls. Fertility is decreasing in the rate of growth of wages. Thus, whether an increase in wage growth (i.e., an acceleration of industrialization) increases or decreases fertility is completely determined by this assumption. Also

\footnotetext{
${ }^{3}$ See Topkis (1998), Chapter 2, for the equivalence between complements/substitutes in utility and the Marshallian definition of complements/substitutes.
} 
clear is that this effect is quite general - it is not restricted to changes in the rate of growth of wages.

I. If $U_{1}>0$, increasing $U_{1}$ increases the marginal utility of children, $M U_{n}$. Because of this, anything that increases $U_{1}$ will lead to a greater desire for children (unless something else changes to offset that).

II. If $U_{1}<0$, increasing $U_{1}$ (smaller number in absolute value) lowers $M U_{n}$, making larger family sizes LESS desirable. Again, this is all holding everything else equal.

From this discussion, we can also get a sense about how changes in the survival rates of children depend on this choice. It is common in the literature (e.g., Barro and Becker (1989), Doepke (2005), Bar and Leukhina (2006)) to model this as a reduction in the cost of producing a surviving child - a reduction in $\theta_{0}$. Notice that this will also typically increase $U_{1}$. A decrease in $\theta_{0}$ causes the left hand side of the equation to shift down. Hence, when children and their utility are complements $\left(U_{1}>0\right)$, it follows that fertility will increase. In the opposite case $\left(U_{1}<0\right)$, there are off-setting effects and the sign of the change cannot be predicted without more detailed analysis. This discussion is complicated by the fact that $U_{1}$ is not exogenous. Because of this, more work is needed to generalize these intuitions below. Finally, note that in this simple case, the parent has no direct method of affecting $U_{1}$, and hence, although one part of a quality/quantity trade-off is present - the direct preference part - other aspects of it are missing (e.g., increasing $U_{1}$ through leaving a larger bequest or spending more on the education of the child). This is a weakness in some ways, but allows us to focus our attention on the importance of the role of preferences much more transparently. ${ }^{4}$

Summarizing then, we see that which assumption we make about whether children and their utility are substitutes or complements - and with it the implicit assumptions about both the sign of $U$ and the monotonicity of $g$ - has important qualitative implications about the properties of the model. As we will see below, almost all work on the Barro-Becker model to date has focused on the first case and this has had important implications about quantitative work using these preferences.

To the best of our knowledge, there is no good evidence for assuming that number and well-being of children are complements. Instead, we find that with low elasticity and substitutability, the model is able to generate trends in fertility similar to those observed over the past 200 years in response to observed changes in mortality, longevity and productivity growth rates. These are also the drivers most emphasized by demographers.

To formally address the effect on fertility choice of these drivers, we introduce both child mortality and adult longevity as follows. First, going back to the original Barro-Becker work one common exercise has been to examine the implications for the model quantities when survival rates of children (e.g., Infant Mortality Rates (IMR) and Child Mortality Rates (CMR)) change. This has been done by assuming

\footnotetext{
${ }^{4}$ In Section 7.2, we address extensions in which the parent has some control over children's utility.
} 
parents care about surviving children, $n_{s, t}=\pi_{s} n_{b, t}$, where $\pi_{s}$ is survival rate of a birth to adulthood and $n_{b, t}$ is the number of births. Note that every birth may be costly even if the child does not survive to adulthood. The total cost of all births is then given by $\theta_{b, t} n_{b, t} .{ }^{5}$ Second, while the usual Barro-Becker model has adults living for only one period, we introduce longevity again using certainty equivalence to keep the state space minimal. This is similar to Blanchard (1985) who laid out the perpetual youth idea. That is, adults survive from one period to the next with a fixed probability $\pi<1$. Longevity conditional on surviving to adulthood is then given by $\sum_{t=0}^{\infty} \pi^{t}=\frac{1}{1-\pi}$ periods, or, if a period last for $T$ years, $\frac{T}{1-\pi}$ years. In the original Barro-Becker model, $\pi=0$ and expected adult lifetime is $T$ years. We then assume that preferences of households are given by ${ }^{6}$

$$
U_{t}=u\left(c_{t}\right)+\beta g\left(\pi+\pi_{s} n_{b, t}\right) U_{t+1}
$$

while the household's budget constraint becomes

$$
c_{t}+\theta_{b, t} n_{b, t} \leq w_{t}
$$

That is, we assume that people care about expected family size and that all adults are identical, regardless of when they were born. Own survival is a perfect substitute for surviving children in the utility. However, survival of both adults $(\pi)$ and children $\left(\pi_{s}\right)$ are exogenous. ${ }^{7}$ Setting $\pi=0$ brings us back to the original Barro-Becker type preference. If adults survival is certain, $\pi=1$, then people live forever but care about themselves exactly as much as they care about each child. Notice that all the results derived above go though with this change. One additional effect is that increased longevity (i.e. $\pi$ ) decreases the marginal utility of having an extra child, regardless of the sign of $U_{t+1}$.

\subsection{Dynasty utility and intertemporal elasticity}

Note that by construction, the preferences given in this way have a natural time consistency property: there is no inherent conflict in preferences between the agents in period $t$ and period $t+1$. In the discussion above it can be seen that to fully specify the model we need an explicit mechanism for the determination of the utility of subsequent generations. This can be done through successive substitution into the utility function of the time 0 agent, the dynasty head to get:

$$
U_{0}=\sum_{t=0}^{\infty} \beta^{t}\left[\Pi_{k=0}^{t-1} g\left(\pi+\pi_{s} n_{b, k}\right)\right] u\left(c_{t}\right)
$$

\footnotetext{
${ }^{5}$ This was used in the original Barro and Becker (1988) and Becker and Barro (1989) as well as in Boldrin and Jones (2002), Schoonbroodt (2004), Doepke (2005), etc. Further layers of complexity are introduced in Section 4.3.2.

${ }^{6}$ Note that with $g($.$) defined over surviving parent plus children, this is similar to Remark 1$ in Benhabib and Nashimura (1993) with $z>0$ interpreted as adult survival.

${ }^{7}$ See Section 7 for issues related to this exogeneity assumption.
} 
Because of the term $\Pi_{k=1}^{t-1} g\left(\pi+\pi_{s} n_{b, k}\right)$, this utility function is typically not concave as written. However, as discussed in Alvarez (1999), under certain conditions, this can be rewritten as a concave problem in dynasty aggregate variables. Assume that $g(x)=x^{\eta}$, and let $N_{0}=1$ and $N_{t}=\Pi_{k=0}^{t-1}\left(\pi+\pi_{s} n_{b, k}\right)$. This is the expected total number of adults (parent and descendants) alive during period $t$ evaluated in $t-1$. Assuming certainty equivalence/law of large numbers, we get the following law of motion for population:

$$
N_{t+1}=\pi N_{t}+N_{s, t}=\Pi_{k=0}^{t}\left(\pi+\pi_{s} n_{b, k}\right)
$$

where $N_{s, t}=n_{s, t} N_{t}$ is the total number of surviving children born in period $t$. Then $\Pi_{k=1}^{t-1} g\left(\pi+\pi_{s} n_{b, k}\right)=g\left(\Pi_{k=1}^{t-1}\left(\pi+\pi_{s} n_{b, k}\right)\right)=g\left(N_{t}\right)$, and so preferences for the dynasty head can be rewritten as:

$$
U_{0}=\sum_{t=0}^{\infty} \beta^{t} g\left(N_{t}\right) u\left[\frac{C_{t}}{N_{t}}\right]
$$

where $C_{t}=N_{t} c_{t}$ is total consumption in period $t$. Note that this assumes that consumption is the same for all adults in a period. $U_{t}$ for $t>0$ is defined similarly.

Following the discussion above, since $g(N)=N^{\eta}$ is always positive, there are two possible ways to satisfy conditions (1.)-(4.) above:

I. Assume that $u(c) \geq 0$ for all $c \geq 0$, that $u$ is strictly increasing and strictly concave and that $0<\eta<1$;

II. Assume that $u(c) \leq 0$ for all $c \geq 0$, that $u$ is strictly increasing and strictly concave and that $\eta<0$.

Either of these are consistent with the entire set of intuitive requirements laid out in the original Barro-Becker papers. Typically we want more however. The extra desirable requirements are that $U_{0}$ as written here is increasing and concave in $(C, N)$. This is for standard reasons, we want the solution to maximization problems to be unique, etc. For this, we specialize further and assume that and $u(c)=\frac{c^{1-\sigma}}{1-\sigma}$. Given this assumption, there are two sets of parameter restrictions that satisfy the natural monotonicity and concavity restrictions for this functional form, both in terms of the aggregate, or dynasty variables, $(C, N)$, and in terms of per capita values, $(N, c)=\left(N, \frac{C}{N}\right)$.

I. The first is the standard assumption in the fertility literature:

$$
\begin{aligned}
& 0 \leq \eta+\sigma-1<1 \\
& 0<1-\sigma<1
\end{aligned}
$$

In this case, $0<\eta=\eta+\sigma-1+1-\sigma<1$ and $U>0$ for all $(N, C) \in R_{+}^{2}$.

II. The second possibility is one which allows for intertemporal elasticities of substitution in line with the standard growth and business cycle literature:

$$
\begin{aligned}
& \sigma>1 \\
& \eta+\sigma-1 \leq 0
\end{aligned}
$$

In this case, $\eta \leq 1-\sigma<0$ and utility is negative. 
In the case where, $\eta=1-\sigma$ (allowed under both configurations), utility becomes a function of aggregate consumption only. ${ }^{8}$ Hence, conditions for monotonicity and concavity of $U$ involve $U_{C}$ and $U_{C C}$ only. ${ }^{9}$

\section{A simple model of fertility choice}

In this Section, we setup the planner's problem with endogenous fertility choice that we study and present analytical comparative statics results across Balanced Growth Paths (BGP's) for each of the parameter configureations laid out above. We begin by focusing only on population growth rates, the natural construct that comes out of BGP's in this type of model. Next, to make comparisons with data easier, we discuss how to construct (surviving) Crude Birth Rates $\left(C B R_{s}\right)$ from the model and give comparative statics results for this measure of fertility. Finally, we discuss the relationship between births and surviving children when infant and child mortality rates are changed. Along with this we introduce a distinction between total births in a period as measured by $C B R$ and the number of surviving births from a period, $C B R_{s}$.

\subsection{The planner's problem}

The model we study is similar in spirit to Becker and Barro (1988) and Barro and Becker (1989). As opposed to Barro-Becker, we allow for a wider preference parameter configuration as described above. Furthermore, we assume that the only source of income is labor and there is no possibility of bequests (see also Doepke (2005)). This allows us to derive clean analytical results. We follow the approach from Alvarez (1999) in which a time zero dynastic head chooses the time paths of aggregate, dynasty level variables. The problem solved is:

$$
\begin{aligned}
& \max _{\left\{C_{t}, N_{b, t}, N_{s, t}, N_{t}\right\}} \quad \sum_{t=0}^{\infty} \beta^{t} N_{t}^{\eta}\left[\frac{C_{t}}{N_{t}}\right]^{1-\sigma} /(1-\sigma) \\
& \text { s.t: } \\
& C_{t}+\theta_{b, t} N_{b, t} \leq w_{t} N_{t}, \text { and } \\
& N_{t+1} \leq \pi N_{t}+N_{s, t} \\
& N_{s, t} \leq \pi_{s} N_{b, t} \text {, } \\
& N_{0} \text { given. }
\end{aligned}
$$

\footnotetext{
${ }^{8}$ The case with parameters in case II. is also mentioned as a possibility in Alvarez (1999), footnote 2, and used in the quantitative section of Mateos-Planas (2002). He uses $\sigma=3$. Although this formulation is not common in the fertility literature, a similar formulation for utility, where the arguments are consumption and leisure, is quite common in the growth literature. There are numerous examples of this, see Ales and Maziero (2007) for a recent example of this. A few remarks and issues with this utility formulation are addressed in the Appendix.

${ }^{9}$ Note that the intertemporal elasticity of substitution in consumption is only partially expressed in $\sigma$. The actual elasticity also involves $N_{t}$. In the case where $\eta=1-\sigma$, the analogy is exact. We will nevertheless stick to this abuse of language for the remainder of the paper.
} 
where $C_{t}$ is aggregate consumption in period $t, N_{t}$ is the number of adults alive, $\theta_{b, t}$ is the cost of a birth, $N_{b, t}$ is the total number of births in period $t, N_{s, t}$ is the number of children that survive to working age and $\pi_{s}$ is the unconditional probability of a child that is born surviving to working age, $N_{s, t}$ is the number of births in period $t$ that survive to become adults in period $t+1$ and $w_{t}$ is the wage rate. ${ }^{10}$ We assume that adults survive to the next period of life with probability $\pi$ but that all alive adults are otherwise identical. Thus, death among adults is treated as in Blanchard (1985). Given these assumptions, the expected working lifetime of an adult is $\frac{1}{1-\pi}$ periods. ${ }^{11}$ Also implicit in this formulation is the assumption that each adult has the same level of consumption $\frac{C_{t}}{N_{t}}=c_{t}$ in any period. This problem can be rewritten by eliminating $N_{b, t}$ to obtain:

$$
\begin{array}{ll} 
& \max _{\left\{C_{t}, N_{s, t}, N_{t}\right\}} \quad \sum_{t=0}^{\infty} \beta^{t} N_{t}^{\eta}\left[\frac{C_{t}}{N_{t}}\right]^{1-\sigma} /(1-\sigma) \\
& C_{t}+\theta_{s, t} N_{s, t} \leq w_{t} N_{t}, \text { and } \\
& N_{t+1} \leq \pi N_{t}+N_{s, t}, \\
& N_{0} \text { given. }
\end{array}
$$

where $\theta_{s, t} \equiv \frac{\theta_{b, t}}{\pi_{s}}$ is the cost of producing a surviving child. ${ }^{12}$ This is formally equivalent to the problem above but where, the cost of raising a child to working age depends on the survival probability - an increase in $\pi_{s}$ decreases $\theta_{s, t}$.

Under either of the sets of parameter restrictions derived in Section 3, this (time zero) maximization problem has a concave objective function and a convex constraint set. Thus, the problems have unique solutions, concave value functions, etc.

\subsection{Equilibrium populations}

The first order condition for the stock of population in period $t+1, N_{t+1}$, is given by:

$$
\begin{aligned}
\theta_{s, t} N_{t}^{\eta+\sigma-1} C_{t}^{-\sigma} & \\
& =\underbrace{\beta\left[w_{t+1}+\theta_{s, t+1} \pi\right] N_{t+1}^{\eta+\sigma-1} C_{t+1}^{-\sigma}}_{A}+\underbrace{\beta \frac{(\eta+\sigma-1)}{(1-\sigma)} N_{t+1}^{\eta+\sigma-2} C_{t+1}^{1-\sigma}}_{B}
\end{aligned}
$$

The intuition for this is as follows. On the left is the marginal cost in terms of changed current utility of increasing $N_{t+1}$ (i.e., of producing an extra child). This

\footnotetext{
${ }^{10}$ Throughout we only look at the 'goods costs case' but this is without loss of generality. This goods cost is denoted by $\theta_{t}$ and we assume that it grows at the rate of overall technological improvement. In the end, the only model object that ends up mattering is $\frac{\theta_{t}}{w_{t}}$ which is constant by assumption. If on the other hand, there was a time cost, say $b$ fraction of the time available in a period per child, we would find that $b=\frac{\theta}{w}$. So everything would be covered in that case as well.

${ }^{11}$ The original Barro-Becker formulation has $\pi=0$. The formulation here has the advantage of allowing us to perform comparative statics with respect to longevity and to calibrate to reasonable life lengths without adding additional state variables.

${ }^{12}$ We introduce additional layers of complexity in Section 4.3.2.
} 
cost is just the direct cost of reduced consumption today (rescaled by the fact that it only takes $\theta_{s, t}$ units of $C$ to make one extra unit of $N$ ). On the right hand side are the two pieces of the marginal benefits next period from increasing $N_{t+1}$. These are: $A$. the value of the extra output the dynasty will have next period; $B$. the marginal value of utility from having extra children.

To gain some more insight, consider the special case in which $\eta=1-\sigma$. In this case, $N$ is exactly like a capital good since the two utility effects of increasing $N$ exactly cancel out. These two effects are: 1 . the direct benefit of having extra children $-\frac{g(N)}{1-\sigma}=\frac{N^{\eta}}{1-\sigma}-$ in the utility function; 2 . the direct cost of having children by diluting per capita consumption $-\frac{\left[\frac{C}{N}\right]^{1-\sigma}}{1-\sigma}-$ in the utility function. As we can see in the first order condition in (2), there are two things that give simplification. The first is that $(\eta+\sigma-1)=0$ and so term (B) disappears entirely and second that $N_{t}^{\eta+\sigma-1}=N_{t+1}^{\eta+\sigma-1}=1$, i.e., the marginal value of increased total consumption by the dynasty in periods $t$ and $t+1$ no longer depend on the size of the dynasty in the period. Because of this, we get:

$$
\left[\frac{C_{t+1}}{C_{t}}\right]^{\sigma}=\beta\left[\frac{w_{t+1}}{\theta_{s, t}}+\frac{\theta_{s, t+1}}{\theta_{s, t}} \pi\right]
$$

This is the standard Euler Equation from an $A k$ model in terms of aggregate consumption, modified for the case $\theta_{s}$ potentially different from 1 with $(1-\pi)$ corresponding to depreciation, and time varying costs and benefits, i.e., $w_{t}$ and $\theta_{s, t}$.

The Euler equation in (2) or (3) together with the feasibility constraint

$$
C_{t}+\theta_{s, t}\left[N_{t+1}-\pi N_{t}\right]=w_{t} N_{t}
$$

which can be rewritten as

$$
\frac{C_{t}}{N_{t}}+\theta_{s, t}\left[\frac{N_{t+1}}{N_{t}}-\pi\right]=w_{t}
$$

and the initial condition $N_{0}$ completely describe the equilibrium path.

\subsection{Balanced growth and comparative statics}

One advantage of the version of the model with labor income only is that it delivers simple, and qualitatively reasonable analytic comparative statics results across Balanced Growth Paths (BGPs). ${ }^{13}$ These are summarized here for completeness. We focus on three distinct changes based on the quantitative experiments that we explore in the next section, namely the results of: i) changing $\gamma$, ii) changing $\theta_{s}$, and iii) changing $\pi$. These correspond to three commonly discussed quantitatively important changes in demographic patterns over the period from 1800 to 1990: i) the

\footnotetext{
${ }^{13}$ Some, but not all of these results carry over to generalized versions of the model with capital. See Section 7.2 for a more detailed discussion.
} 
increased growth rate of labor productivity that came with industrialization, ii) the fall in the direct cost of creating a surviving child through the reduction in infant and child mortality rates, and iii) the substantial increase in expected lifetime that has occurred in the last 100 years. As a first approximation, we study these changes by deriving simple comparative statics with respect to the parameters of the model.

Assume that $\theta_{s, t}=\gamma^{t} \theta_{s}$ and $w_{t}=\gamma^{t} w$. Let $\gamma_{N, t}=\frac{N_{t+1}}{N_{t}}$ be the population growth rate between $t$ and $t+1$, let $\gamma_{C}=\frac{C_{t+1}}{C_{t}}$ be the growth rate in aggregate consumption and let $\gamma_{c}=\frac{C_{t+1} / N_{t+1}}{C_{t} / N_{t}}$ be the growth rate in per capita consumption. On a BGP, the resource constraint in (4) becomes

$$
\frac{1}{\gamma^{t}} \frac{C_{t}}{N_{t}}=w+\theta_{s} \pi-\theta_{s} \gamma_{N}
$$

Since on a BGP (5) has to hold for every $t$, we must have

$$
\frac{C_{t}}{\gamma^{t} N_{t}}=\frac{C_{t+1}}{\gamma^{t+1} N_{t+1}} \quad \text { or } \quad \gamma=\frac{C_{t+1} / N_{t+1}}{C_{t} / N_{t}}=\frac{\gamma_{C}}{\gamma_{N}}=\gamma_{c}
$$

Then, using this and (5) in (2) after dividing both sides by $\gamma^{t+1} N_{t+1}^{\eta+\sigma-1} C_{t+1}^{-\sigma}$ and rearranging, we get

$$
\frac{1}{\beta} \gamma_{N}^{1-\eta} \gamma^{\sigma-1}+\frac{(\eta+\sigma-1)}{(1-\sigma)} \gamma_{N}=\frac{\eta}{(1-\sigma)}\left[\frac{w}{\theta_{s}}+\pi\right]
$$

\subsubsection{Comparative statics of population growth}

The only endogenous variable in equation (6) is the population growth rate, $\gamma_{N}$, which only enters on the left-hand side. Moreover, $\pi$ (expected working life) and $\theta_{s}$ (costs of children or youth mortality) only enter on the right-hand side while the productivity growth rate, $\gamma$, only enters on the left-hand side. That is, holding $(\sigma, \beta, \eta, w)$ fixed, this equation is of the form: $\operatorname{LHS}\left(\gamma_{N} ; \gamma\right)=D\left(\theta_{s}, \pi\right)$, where $D\left(\theta_{s}, \pi\right)=\frac{\eta}{(1-\sigma)}\left[\frac{w}{\theta_{s}}+\pi\right]$ (see Figure 2). Note that $\operatorname{LHS}\left(\gamma_{N} ; \gamma\right)$ is increasing in $\gamma_{N}$, for all values of $\gamma$, since in both parameter restrictions we have that $\eta \in[-\infty, 1)$ and $\frac{(\eta+\sigma-1)}{(1-\sigma)}>0$. Similarly, $D>0$ since $\frac{\eta}{(1-\sigma)}>0$.

Because of this, it follows that $\operatorname{LHS}\left(\gamma_{N} ; \gamma\right)$ is increasing in $\gamma^{\sigma-1}$ holding $\sigma$ fixed. However, whether an increase in $\gamma$ causes $\gamma^{\sigma-1}$ to increase or decrease depends on whether $\sigma>1$ or $\sigma<1$. If $\sigma<1$, an increase in $\gamma$ causes $\gamma^{\sigma-1}$ to fall while if $\sigma>1$, an increase in $\gamma$ causes $\gamma^{\sigma-1}$ to rise. From this it follows that $\gamma_{N}$ is increasing in $\gamma$ if $\sigma<1$ and decreasing in $\gamma$ if $\sigma>1$. The result that the sign intuition for this result comes from the fact that for low (high) IES children and their utility are substitutes (complements) in utility as shown in Section 3. However, the desire to smooth consumption is important for the size of this effect.

$D$, on the other hand, is increasing in $\pi$ and decreasing in $\theta_{s}$ for either parameter configuration, and hence so is $\gamma_{N}$. Again, we can derive results about the size of effects of changes in parameter values on $\gamma_{N}$ for different values of $(\eta, \sigma)$. To see this, 
Figure 2: Comparative statics of $\gamma_{N}$

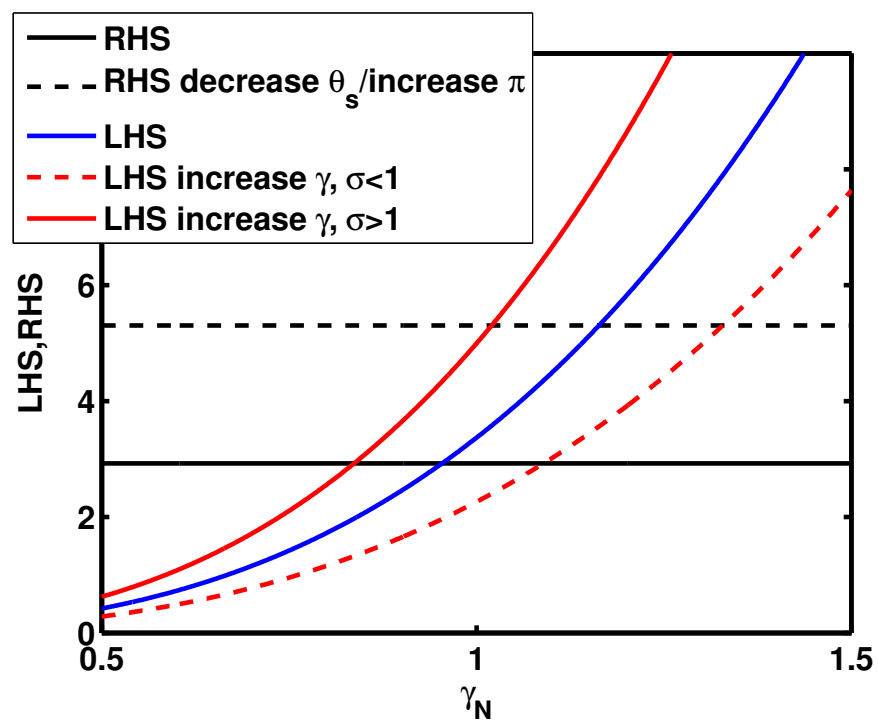

Figure 3: Increase in $\gamma_{N}$ is smaller for $\eta \leq 1-\sigma<0$ than for $\eta \geq 1-\sigma>0$

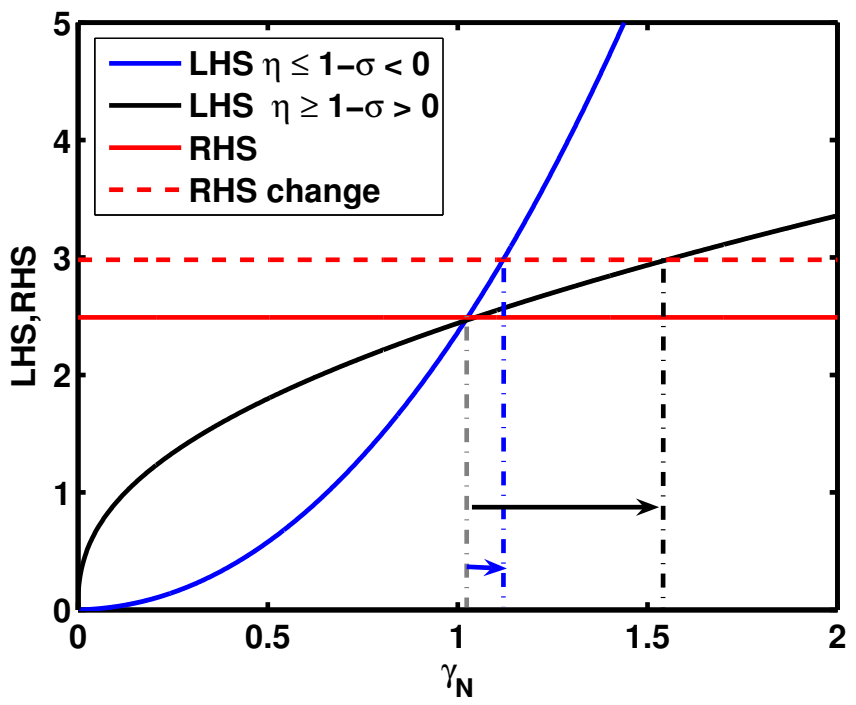


note that the left-hand side of equation (6) is increasing in $\gamma_{N}$. Further, it is concave if $1>\eta \geq 1-\sigma>0$ (i.e. $\sigma<1$ ), and convex in $\gamma_{N}$ if $\eta \leq 1-\sigma<0$ (i.e. $\sigma>1$ ) (see Figure 3). Because of this, it follows that if $\gamma_{N}>1$, a given size change in $\theta_{s}$, or $\pi$ has larger effects on $\gamma_{N}$ in the low curvature (high IES) case.

Further, equation (6) simplifies considerably when $1-\sigma=\eta$. In fact,

$$
\gamma_{N}^{\sigma}=\beta \gamma^{1-\sigma}\left[\frac{w}{\theta_{s}}+\pi\right]
$$

We use this version of the Euler equation in our quantitative analysis below. Summarizing the discussion to here we see:

Proposition 1. The following comparative statics results across BGP's hold for population growth, $\gamma_{N}$ :

1. An increase in $\gamma$ causes $\gamma_{N}$ to rise if $1>\eta \geq 1-\sigma>0$ and to fall if $\eta \leq$ $1-\sigma<0$.

2. An increase in the cost of children, $\theta_{s}$, causes the population growth rate, $\gamma_{N}$ to fall.

3. An increase in adult survival, $\pi$, causes $\gamma_{N}$ to increase.

Moreover, if $\gamma_{N}>1$, a change in $\theta_{s}$ or $\pi$ has larger effects on the BGP level of $\gamma_{N}$ if $1>\eta \geq 1-\sigma>0$ than if $\eta \leq 1-\sigma<0$.

It also follows that $\frac{\partial \gamma_{N}}{\partial \beta}>0$ and $\frac{\partial \gamma_{N}}{\partial w}>0$ if $\theta_{s}=a$ (goods cost) and $\frac{\partial \gamma_{N}}{\partial w}=0$ if $\theta_{s}=b w$ (time cost). We do not emphasize these because they play no role in the quantitative discussion we focus on below. ${ }^{14}$

These comparative statics have important implications for studying trends in fertility using this type of model. From the beginning of the 19th century to the end of the 20th century, Crude Birth Rates (CBR) fell substantially, while population growth rates (net of immigration) decreased only slightly. Since the rate of growth of productivity has increased over the period describing these demographic changes, it follows from this that we would expect population growth rates to fall as a result as long as $\sigma>1$. We explore this issue quantitatively below. Moreover, note that a decrease in youth mortality is interpreted as a decrease in the cost of surviving children, $\theta_{s}$, while increases in expected working life lengths are interpreted as increases in $\pi$. Hence, smaller increases in population growth in response to changes in mortality declines (through $\theta_{s}$ or $\pi$ ) may be a desirable prediction. From the proposition, this is the case with lower IES (i.e., $\sigma>1$ ). In the next two subsections, we derive a model analogue for the Crude Birth Rate and introduce survival probabilities to adulthood to discuss relevant comparative statics.

\footnotetext{
${ }^{14}$ If all child costs are goods costs, increases in labor income taxes are equivalent to reductions in $w$. Thus, it follows that increasing the labor income tax rates will decrease both population growth rates and fertility on the BGP, cf. Manuelli and Seshadri (2005). At the other extreme, if all costs are in terms of time $(\theta=b w)$, fertility and population growth rates are independent of labor income taxes.
} 


\subsubsection{Comparative statics of the Crude Birth Rate, surviving children}

In this version of the model, the variable of choice is the total number of surviving births in the dynasty in a given period, $N_{s, t}$. This does not map naturally to the usual measures of fertility used by demographers such as Total Fertility Rate (TFR) or Cohort Total Fertility Rate (CTFR). This is complicated by the fact that the model is unisex and monoparental - i.e., each agent has children and is on his/her own and this is true for everyone, not just women. These assumptions are made for simplicity. ${ }^{15}$ There is a natural relationship, however, between $N_{s, t}$ and a common measure of fertility, the Crude Birth Rate-CBR. In the data, the latter is calculated as the number of children born in a period divided by the population in that period. In the model, we have:

$$
C B R_{s, t}=\frac{N_{s, t}}{N_{t}+N_{s, t}}=\frac{N_{t+1}-\pi N_{t}}{N_{t}+N_{t+1}-\pi N_{t}}=\frac{\gamma_{N t}-\pi}{1+\gamma_{N t}-\pi},
$$

where $\gamma_{N t}=\frac{N_{t+1}}{N_{t}}$ is the growth rate of the adult population between periods $t$ and $t+1$. This expression for $C B R_{s}$ corresponds to the number of births during a period divided by the end of period population. Here, we are assuming that all children survive giving the expression in the text. Below, we also introduce infant and child mortalities and differentiate between CBR measured in births vs. CBR measured in surviving children. Thus, this is one obvious identification between data and model quantities that can be used.

On the BGP, $C B R_{s}$ is constant and is given by:

$$
C B R_{s}=\frac{\gamma_{N}-\pi}{1+\gamma_{N}-\pi}=\frac{1}{1+\frac{1}{\gamma_{N}-\pi}} .
$$

This is of the form $\frac{1}{1+1 / x}$ and because of this it follows that the comparative statics results given above for $\gamma_{N}$ for changes in $\gamma$ and $\theta_{s}$ also hold for $C B R_{s}$. The one exception to this concerns the effects of changes in expected life length. Even in this case, $C B R_{s}$ is a monotonically increasing function of $\left(\gamma_{N}-\pi\right)$, and so the sign of $\frac{\partial C B R_{s}}{\partial \pi}$ is the same as the sign of $\frac{\partial \gamma_{N}}{\partial \pi}-1$. Thus, while an increase in $\pi$ always increases population growth rates, whether or not it increases $C B R_{s}$ depends on the size of $\frac{\partial \gamma_{N}}{\partial \pi}$. If it is less then $1, \frac{\partial C B R_{s}}{\partial \pi}<0$ while the opposite holds if $\frac{\partial \gamma_{N}}{\partial \pi}>1$. When $\eta=1-\sigma$, we find that:

$$
\frac{d \gamma_{N}}{d \pi}=\frac{\beta\left(\gamma \gamma_{N}\right)^{1-\sigma}}{\sigma}
$$

Note that $\gamma \gamma_{N}=\gamma_{C}$, the growth rate of aggregate consumption by the dynasty. As is standard in growth models, $\beta\left(\gamma \gamma_{N}\right)^{1-\sigma}$ is the growth rate in utility. This must be less than one for a well defined (finite) solution to exist for the dynasty's maximization problem. If $\sigma>1$, that condition holds if $\beta<1, \gamma>1$ and $\gamma_{N} \geq 1$.

\footnotetext{
${ }^{15}$ See Section 7 for further discussion of other measures of fertility.
} 
Thus, it follows that in this case, $\frac{\partial C B R_{s}}{\partial \pi}<0$ as long as $\gamma_{N} \geq 1$. If $\sigma<1$, the condition is $\beta\left(\gamma \gamma_{N}\right)^{1-\sigma}<\sigma$ which is not necessarily satisfied. Summarizing, we have:

Proposition 2. The following comparative statics results hold across BGP's for surviving children, $C B R_{s}$ :

1. An increase in $\gamma$ causes $C B R_{s}$ to rise if $1>\eta \geq 1-\sigma>0$ and to fall if $\eta \leq 1-\sigma<0$.

2. An increase in the cost of children, $\theta_{s}$, causes the fertility rate, $C B R_{s}$ to fall.

3. If $\eta=1-\sigma<0$, and $\beta \gamma^{1-\sigma}\left[\frac{w}{\theta_{s}}+\pi\right]>1$ (i.e., $\gamma_{N}>1$ ), an increase in $\pi$ causes $C B R_{s}$ to fall.

Moreover, if $\gamma_{N}>1$, a change $\theta_{s}$ has larger effects on the $B G P$ level of $C B R_{s}$ if $1>\eta \geq 1-\sigma>0$ than if $\eta \leq 1-\sigma<0$.

For the quantitative assessments below, it is interesting to note that by analogy to a growth model, although a decrease in the depreciation rate causes the rate of growth of the capital stock (i.e., population growth in this interpretation) to increase, this does not necessarily imply that investment's share in output (i.e., fertility) increases. Indeed, when $\eta=1-\sigma<0$, population growth, $\gamma_{N}$, increases while fertility, $C B R_{s}$, falls in response to an increase in $\pi$.

\subsubsection{The effects of changes in survival to adulthood: births vs. survivors}

Going back to the original Barro-Becker work one common exercise has been to examine the implications for the model quantities when survival rates of children (e.g., Infant Mortality Rates (IMR) and Child Mortality Rates (CMR)) change. ${ }^{16}$ Because of the equivalence of the problems derived in Section 4, an increase in $\pi_{s}$ lowers the cost of children and hence, by the argument above increases $\gamma_{N}$ and $C B R_{s}$. Note that it is $\gamma_{N}$ and $C B R_{s}$ calculated in terms of surviving children that increase and this does not necessarily imply that, for example, $C B R$ calculated in terms of births goes up. To make this distinction clear we introduce one new piece of notation

$$
C B R_{t}=\frac{N_{b, t}}{N_{t}+N_{s, t}}=\frac{C B R_{s, t}}{\pi_{s}}
$$

Thus, even though $C B R_{s}$ is increasing in $\pi_{s}$ it need not be true that $C B R$ is. Also, the smaller the increase in $C B R_{s}$ the larger the decrease (smaller the increase) in $C B R$ in response to an increase in youth survival $\pi_{s}$. This is the case for low levels of IES as per Proposition 2.

Additional layers of complexity can also be added (cf. Doepke (2005) for examples). For example suppose that there are 3 stages that children must pass through to become adults, say, infancy, childhood and youth. Suppose i) $\pi_{i}$ is the survival probability of infants which $\operatorname{cost} \theta_{i}$ to raise ii) $\pi_{i c}$ is the conditional survival probability to

\footnotetext{
${ }^{16}$ This was used in the original Barro and Becker (1988) and Becker and Barro (1989) as well as in Boldrin and Jones (2002), Schoonbroodt (2004), Doepke (2005), etc.
} 
be a youth given survival through infancy and $\theta_{c}$ is the cost borne by parents during childhood, and iii) $\pi_{c y}$ is the probability of surviving to adulthood conditional on surviving to be a youth and $\theta_{y}$ is the cost to parents of children in youth. Then the total child-rearing cost to a parent that has $N_{b}$ births is $\theta_{i} N_{b}+\theta_{c} \pi_{i} N_{b}+\theta_{y} \pi_{i c} \pi_{i} N_{b}$ while the total number of surviving children produced is $N_{s}=\pi_{s} N_{b}$, where $\pi_{s}=\pi_{i} \pi_{i c} \pi_{c y}$. Thus, to produce $N_{s}$ surviving children, $N_{b}$ must be $\frac{N_{s}}{\pi_{s}}$ and hence, the total cost is:

$$
\theta_{s} N_{s} \equiv\left(\theta_{i}+\pi_{i} \theta_{c}+\theta_{y} \pi_{i c} \pi_{i}\right) N_{b}=\frac{\theta_{i}+\pi_{i} \theta_{c}+\theta_{y} \pi_{i c} \pi_{i}}{\pi_{s}} N_{s}
$$

or, the per surviving child cost is:

$$
\theta_{s}=\frac{\theta_{i}}{\pi_{i} \pi_{i c} \pi_{c y}}+\frac{\theta_{c}}{\pi_{i c} \pi_{c y}}+\frac{\theta_{y}}{\pi_{c y}} .
$$

Again, we see that increasing survival rates $\left(\pi_{i}\right.$ or $\pi_{i c}$ or $\pi_{c y}$ or any subset) lowers the cost of producing a surviving child and hence from the results above both the population growth rate and $C B R_{s}$ increase. As above, this need not be true of $C B R$, however. We use this formulation in our time series experiment for the U.S. in Section 6.

Two special cases are worthy of mention. The first is one in which all costs have to be borne for all births, e.g. $\pi_{i}=\pi_{i c}=1$. In this case we are back to the case described in the beginning of this section where $\pi_{s}=\pi_{c y}$ and $\theta_{b}=\theta_{i}+\theta_{c}+\theta_{y}$. This is the case we consider in our first set of quantitative experiments. The second one is where only survivors are costly, while births that die before a certain age are costless. For example, if $\pi_{i c}=\pi_{c y}=1$ (i.e., children die in infancy or survive to adulthood) and $\theta_{i}=0$, then $N_{s}$ and $C B R_{s}$ are independent of $\pi_{s}=\pi_{i}$. Thus, $N_{b}=\frac{N_{s}}{\pi_{i}}$ and $C B R=\frac{C B R_{s}}{\pi_{i}}$ are clearly decreasing (and hyperbolic) in $\pi_{i}$ (see Doepke (2005) for a full exposition). In general, however, whether the number of births, $C B R$ is increasing or decreasing in $\pi_{s}$ depends on which of the two percentage increase is larger, the one in $C B R_{s}$ or the one in $\pi_{s}$. Again, we summarize in a proposition:

Proposition 3. The following comparative statics results hold across BGP's for total births, $C B R$ :

1. An increase in $\gamma$ causes $C B R$ to rise if $1>\eta \geq 1-\sigma>0$ and to fall if $\eta \leq 1-\sigma<0$.

2. If If $\eta=1-\sigma<0$, and $\beta \gamma^{1-\sigma}\left[\frac{w}{\theta_{s}}+\pi\right]>1$ (i.e., $\gamma_{N}>1$ ), an increase in $\pi$ causes $C B R$ to fall.

Moreover, in both cases, percentage changes in $C B R$ are equal to percentage changes in $C B R_{s}$.

3. An increase in $\pi_{s}$ causes $C B R$ to fall if $\frac{d C B R_{s}}{d \pi_{s}}<\frac{C B R_{s}}{\pi_{s}}$.

As noted in the previous section, it is important for what is coming to remember that although increases in the components of $\pi_{s}$ (and hence decreases in $\theta_{s}$ ) increase $C B R_{s}$, this increase is smaller when $\sigma>1$. Hence, it is more likely that the condition in (3.) holds if $\sigma>1$. 


\section{Quantitative comparative statics}

Our next task is to extend the insights gained above to get quantitative estimates of the model predictions in terms of population growth rate and CBR in response to (unanticipated) changes in the parameters as seen in the data.

First, we discuss how the model can be calibrated to match (current) levels of fertility and/or population growth rates. Most of the parameters needed can be taken directly from the quantitative growth and RBC literatures. The exceptions to this are $\theta$, the cost of raising a child, and $\eta$ the utility parameter governing curvature over dynasty size. For simplicity we set $\eta=1-\sigma$ throughout. ${ }^{17}$ We find that when $\sigma>1$, the needed calibrated costs to match any given fertility level are substantially lower.

Second, we examine the quantitative comparative statics on BGP fertility of (unexpected) changes in i) growth rates in productivity (or wages), ii) survival rates to adulthood, iii) expected working lifetimes as observed in many western countries over the last 200 years. In keeping with the comparative statics results highlighted in the previous section, we find:

i) an increase in the rate of growth of wages causes a substantial reduction in population growth rates when $\sigma>1$, no change when $\sigma=1$ and a significant increase when $\sigma<1 ;^{18}$

ii) an increase in survival to adulthood increases surviving fertility and population growth rates, but this effect is significantly smaller when $\sigma>1$, indeed the number of total births falls substantially;

iii) increasing expected lifetimes increases the population growth rate, but fertility falls, and the size of the fall is larger when $\sigma$ is higher.

In the last section, we combine these three effects to see what the predicted effects on fertility are from changing all three of these factors with the appropriate timing of events in the U.S. over the last 200 years. We find that it predicts that the CBR falls from about 36 to 17 births per 1000 population, capturing two thirds of the 45 to 16 decrease observed in the data and with similar timing.

\subsection{Calibration: fertility levels and costs of children}

In this subsection we use the Euler equation in (7) to calibrate costs of children given all other parameters and targeting zero population growth. This exercise corresponds roughly to choosing a cost of children that matches the fertility experience (i.e., about 15 birth per 1000 population) of the developed countries (e.g., U.K., U.S., Germany, France, etc.) over the 1970 to 1990 period. We find that the cost parameter needed to

\footnotetext{
${ }^{17}$ Note that given our two admissible parameter configurations, $\eta$ would have to be adjusted whenever we compare results for values of $\sigma>1$ and $\sigma<1$. Hence, the assumption that $\eta=1-\sigma$ is fairly innocuous. In this case, the two utility effects of increasing dynasty size cancel out and children are a pure investment good - independently of $\sigma$.

${ }^{18} \mathrm{By} \sigma=1$ we mean the case where $U_{0}=\sum \beta^{t} \log C_{t}$.
} 
match this population growth rate is highly sensitive to our choice of $\sigma$. For example, if $\sigma=0.5$ it is necessary that the cost of a child is about 15.4 years of one adult's output. On the other hand, if $\sigma=3$, this cost drops to about 4.5 years of output per child.

From the results of the previous section, we find:

$$
\gamma_{N}=\left[\beta\left[\frac{w}{\theta_{s}}+\pi\right]\right]^{1 / \sigma}[\gamma]^{1 / \sigma-1}
$$

Now, suppose there are $T$ years in a period. Then we can rewrite the equation above in annual terms as (the subscript ann denotes annual values):

$$
\gamma_{N, a n n}^{T}=\left[\beta_{\text {ann }}^{T}\left[\frac{w}{\theta_{s}}+\pi_{\text {ann }}^{T}\right]\right]^{1 / \sigma}\left[\gamma_{\text {ann }}^{T}\right]^{1 / \sigma-1}
$$

Solving for $\frac{\theta_{s}}{w}$ gives

$$
\frac{\theta_{s}}{w}=\frac{1}{\left[\frac{\gamma_{N, a n n}^{\sigma}}{\beta_{a n n} \gamma_{a n n}^{(1-\sigma)}}\right]^{T}-\pi_{a n n}^{T}} .
$$

where the relevant $\pi_{a n n}^{T}$ is the probability of an adult surviving (in the workforce) for $T$ years. We choose $T=20$ and hence $\pi_{a n n}=0.971$ to match an expected adult (working) life, $\frac{T}{1-\pi}$, of 45 years. Moreover, we assume an annual discount factor of $\beta_{\text {ann }}=0.96$, an annual productivity growth rate of $\gamma_{\text {ann }}=1.02$, an annual population growth of $\gamma_{N, a n n}=1.00$ and zero infant, child and youth mortality, i.e. $\pi_{i}=\pi_{i c}=\pi_{c y}=1.0$ (in line with recent experience in developed countries). Thus, $\theta_{s}=\theta_{b}$ in our calibration. We consider various values of $\sigma \in\{0.5,1,2,3\}$.

Table 1: Costs of children

\begin{tabular}{c|c|c}
\hline \hline$\sigma$ & $\frac{\theta_{s}}{w}$ & $T \times \frac{\theta_{s}}{w}$ \\
\hline \hline & & \\
0.5 & 0.77 & 15.38 \\
1.0 & 0.59 & 11.72 \\
2.0 & 0.36 & 7.13 \\
3.0 & 0.23 & 4.50 \\
\hline \hline
\end{tabular}

The units of $\frac{\theta_{s}}{w}$ are the fraction of one periods per capita output that it costs to raise one child. To get a sense of how costly children must be in the model in order to match realistic growth rates of population, it is more convenient to express it in terms of the number of years of output that are required to raise a child, i.e., $T \times \frac{\theta_{s}}{w}$. Our choice of $T=20$ also corresponds to assuming that it takes 20 years for a newborn 
to become a productive worker. Given this, for various values of $\sigma$, our findings are summarized in Table 1.

Clearly, the values of the costs of raising children - in the range of 5 to 15 years of one person's output - are very sensitive to the choice of the intertemporal elasticity of substitution in consumption (IES). The intuition for this is as follows. When $\sigma$ is high (i.e., there is a strong desire to smooth consumption), high growth in aggregate consumption is not valuable (as above, since the two utility effects of dynasty size cancel out this is the only effect that matters). Aggregate consumption grows at rate $\left(\gamma \gamma_{N}\right)$. Hence, everything else equal, the population growth rate is decreasing in the desire to smooth consumption $(\sigma)$. Vice versa, to get a given population growth rate (i.e., fertility level) to be optimal, one needs higher costs of children when $\sigma$ is lower.

These results have important implications about realism. For example, one implication is that if $\sigma=0.5$, to keep population constant approximately $77 \%$ of each persons output between the ages of 20 and 40 must be devoted to child-rearing, an unrealistically high number. In contrast, when $\sigma=3.0$, the corresponding number is only $23 \%$. $^{19}$

\subsection{Industrialization and the transition to productivity growth}

In this subsection we present the quantitative effect of an increase in the rate of technological progress, $\gamma$, from 0 to 2 percent on the population growth rate (and $\left.C B R_{s}\right)$. We use the calibration of costs from Table 1 so that the zero population growth target, $\gamma_{N, \text { ann }}=1.00$ remains for $\gamma_{a n n}=1.02$. Given our calibration strategy, we assume that $\pi_{i}=\pi_{i c}=\pi_{c y}=1.0$ throughout for this experiment. Because of this, $C B R=C B R_{s} \cdot{ }^{20}$ In Figure 4 (and Table A.1), we report $C B R$ at an annual frequency. This is calculated as:

$$
C B R_{s, a n n}=\frac{C B R_{s}}{T}
$$

In line with comparative statics results in Propositions 1 and 2, we can also see that for high values of IES $(\sigma=0.5), C B R_{s}$ increases by a factor of three while for low values of IES, it decreases by about 25 percent in response to an increase in productivity growth from 0 to 2 percent per year.

\subsection{Survival to adulthood}

Here, we use the calibrated version of the model to assess the impact of increases in survival rates to adulthood that have been observed over the last 150 to 200 years.

\footnotetext{
${ }^{19}$ Equivalently, if $\sigma=0.5$, it is only possible for two parents to raise $2 / 0.77 \approx 3$ children, and that this would require $100 \%$ of both parents efforts. When $\sigma=3.0$, on the other hand, this calculation gives $2 / 0.23 \approx 8.0$ children.

${ }^{20}$ Note that as long as costs of children are adjusted appropriately, the length of the time period does not matter for this result.
} 
Figure 4: $C B R_{s}$ as a function of $\gamma$ for different values of $\sigma$

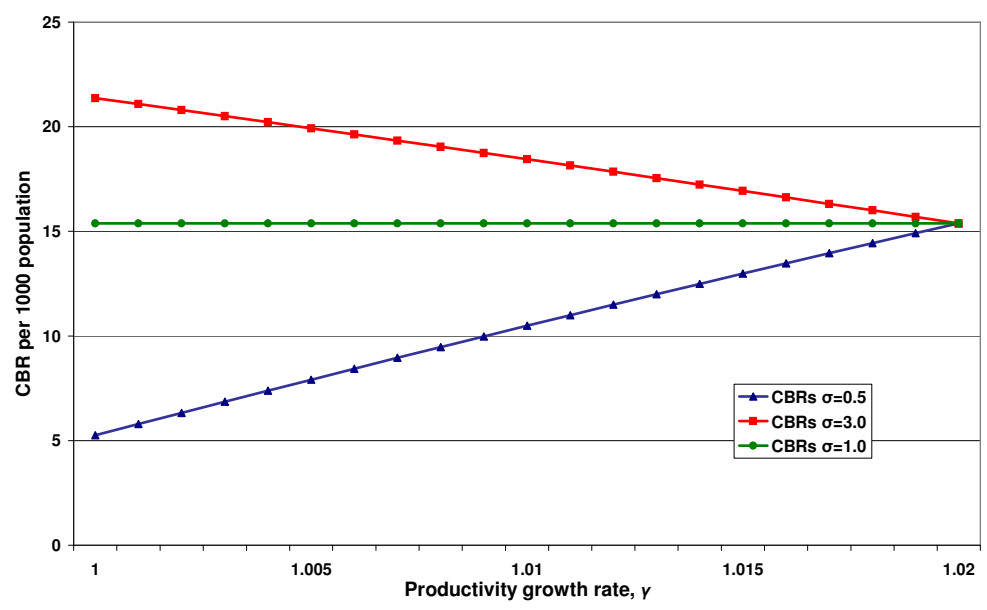

For the most part we use the values from Doepke (2005) to facilitate comparisons and highlight the effects of higher curvature in the utility function.

To do this, we assume, as above, that there are three sub-periods before reaching working age after one period, 20 years. Thus, we need to specify both the subperiod costs, $\left(\theta_{i}, \theta_{c}, \theta_{y}\right)$ and the sub-period conditional survival rates, $\left(\pi_{i}, \pi_{i c}, \pi_{c y}\right)$. We assume that in 1970, $\pi_{i}=\pi_{i c}=\pi_{c y}=1$, and follow Doepke (2005) in assuming that $\theta_{y}=0$ and costs are the same for every year age 0 to 5 , i.e. $\frac{\theta_{c}}{\theta_{i}}=4$. Thus, given this and our finding above on $\theta_{s} / w$ in the calibration section we get $\left(\theta_{i}, \theta_{c}, \theta_{y}\right) / w=$ $\left(\frac{1}{5} \frac{\theta_{s}}{w}, \frac{4}{5} \frac{\theta_{s}}{w}, 0\right)$. Note that these vary with $\sigma$. However, we assume that these costs have not changed over time while mortality rates have - decreasing the effective cost of surviving children.

We consider the following exercise given these cost parameters. First, we assume that $\pi_{i}=\pi_{i c}=1$, but, we vary $\pi_{c y}$ from 0.6 to 1 . This corresponds to a situation in which the full cost up to age 20 must be borne for every birth, but not all births survive to become working adults. Our results are summarized in Figure 5 (and Table A.2).

For all three values of $\sigma$, surviving fertility $\left(C B R_{s}\right)$ increases while total births $(C B R)$ decreases. However, for higher values of $\sigma$ (lower IES) the increase in $C B R_{s}$ is less pronounced while (for this range of $\pi_{c y}$ ) the decrease in $C B R$ is larger. Similar to what we saw with changes in productivity, larger values of $\sigma$ predict changes in fertility and changes in population growth rates that are more closely in line with observed historical trends. In the time series exercise in the next section, we change all three subperiod survival probabilities. 
Figure 5: $C B R$ and $C B R_{s}$ as a function of $\pi_{c y}$ for different values of $\sigma$

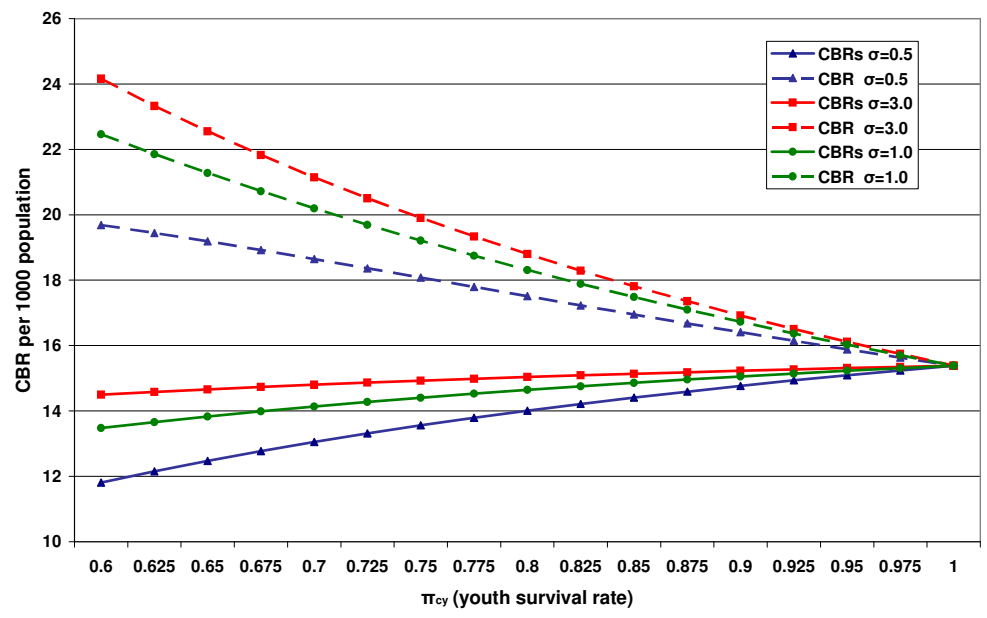

Figure 6: $C B R_{s}$ as a function of $E W L$ for different values of $\sigma$

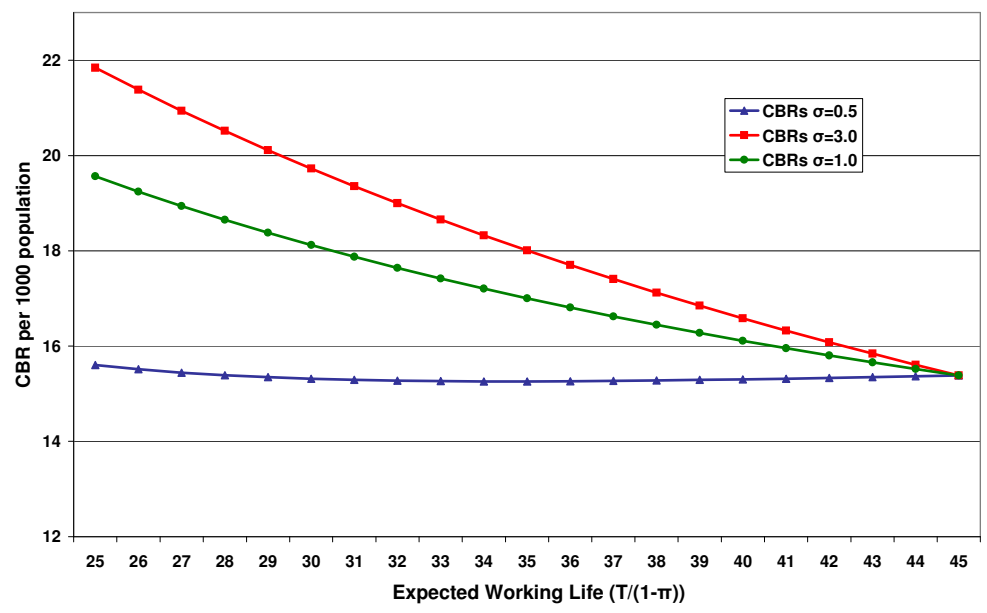




\subsection{Life expectancy}

Here we study the quantitative effects on fertility choice of changing the expected working lifetime of an adult, $\pi$. As above, we use the calibration as our base case with $\pi_{a n n}=0.971$ to match an expected working life of 45 years and calculate the effects of lowering $\pi$ to decrease expected working life to 25 years. As in Section 5.2, note that in this exercise, $C B R=C B R_{s}$ since $\pi_{s}=1$ throughout by assumption. Again, we consider $\sigma=0.5, \sigma=1$ and $\sigma=3.0$. Our results for this case are summarized in Figure 6 (and Table A.3). Expected working life, $\frac{T}{1-\pi}$, is reported in column 2 .

As can be seen in Table A.3, an increase in expected working life increases population growth, $\gamma_{N}$, for all values of IES. $C B R_{s}$ however, decreases, and more so for lower values of the IES. In fact, for $\sigma=0.5$, fertility remains roughly constant, while for $\sigma=3.0$, it decreases by about 30 percent.

\section{The U.S. experience 1800-1990}

The pattern of fertility decline in the U.S. from 1800 to 1990 is as follows. According to Hacker (2003), CBR was roughly constant at 45 births per 1000 population from 1800 to 1860 , then decreased to 19 in $1930 .^{21}$ From Haines (1994b), we find that from 1930, the bottom of the pre WWII baby bust, CBR increased to 23 in the 1950s and 1960s, the peak of the post WWII baby boom, and finally fell to 15.8 in 1990 (Figure 7). ${ }^{22}$ From Haines (1994b), population growth rates (net of immigration), as plotted in Figure 8, decreased from 2.6 percent per year to 0.65 percent per year, again with a down then upward swing from 1930 to 1960.

As a final experiment, we examine the effects of simultaneously changing $\gamma, \theta_{s}$ (through changes in $\pi_{i}, \pi_{i c}$, and $\pi_{c y}$ ) and $\pi$ in line with the experience of the U.S. (and other industrialized countries, see the Appendix for the case of the U.K.) over the period from 1800 to 1990 . We calibrate to the U.S. population growth rate (rate of natural increase, see Haines (1994b), Table 1) in the 1970s of $\gamma_{a n n, N}=1.0065$. This implies costs of children as summarized in Table 2.

As in Section 5.3 we assume that base costs in infancy and childhood are a fraction of calibrated costs when infant and child mortality are zero. In terms of the costs in the table above, we have $\left(\theta_{i}, \theta_{c}, \theta_{y}\right) / w=\left(\frac{\pi_{s}}{1+4 \pi_{i}} \frac{\theta_{s}}{w}, \frac{4 \pi_{s}}{1+4 \pi_{i}} \frac{\theta_{s}}{w}, 0\right)$. We assume that these stay constant throughout the experiment. ${ }^{23}$

We use data on productivity growth, survival probabilities to adulthood and expected lifetimes in the U.S. from 1800 to 1990 (see Table A.4 in the Appendix for the

\footnotetext{
${ }^{21}$ See Table A.4 for further detail. Note that earlier estimates of CBR in the 19th century were higher, at about 55 births per 1000 population in 1800 to 45 in 1850 (e.g. Haines (1994b)).

${ }^{22}$ For an application of tools similar to those used in this paper with stochastic productivity to address fluctuations in fertility, see Jones and Schoonbroodt (2006).

${ }^{23}$ See Mateos-Planas (2002) for an estimation of how these base costs must have changed in several European countries to fully match their fertility experience.
} 
Table 2: U.S. Costs of children in 1990, Time Series Experiment

\begin{tabular}{c|c|c}
\hline \hline$\sigma$ & $\frac{\theta_{s}}{w}$ & $T \times \frac{\theta_{s}}{w}$ \\
\hline \hline 0.5 & 0.72 & 14.32 \\
1.0 & 0.51 & 10.25 \\
3.0 & 0.16 & 3.24 \\
\hline \hline
\end{tabular}

exact time series used and data sources). This data implies the following ranges for $\left(\gamma, \pi_{i}, \pi_{i c}, \pi_{c y}, \pi\right):$

- in $1800,\left(\gamma, \pi_{i}, \pi_{i c}, \pi_{c y}, \pi\right)=(1.00,0.77,0.89,0.92,0.58)$;

- in 1990, $\left(\gamma, \pi_{i}, \pi_{i c}, \pi_{c y}, \pi\right)=(1.018,0.99,1.0,1.0,0.62)$.

Note that, in the model, agents assume that current values of these parameters will prevail forever. ${ }^{24}$

Figure 7: The U.S. experience from 1800 to $1990, C B R$

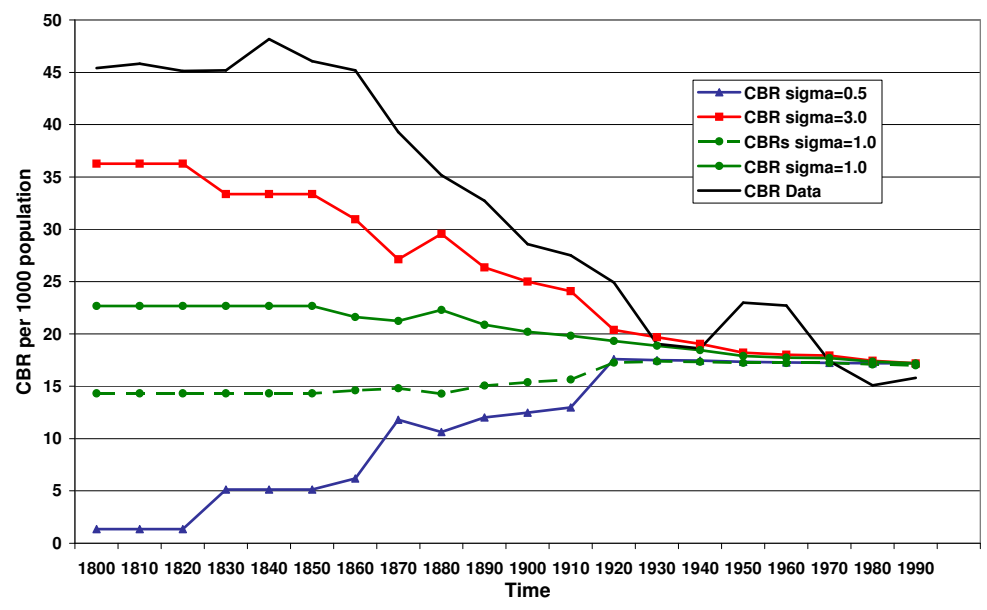

\footnotetext{
${ }^{24}$ The assumption that agents believe that productivity growth, mortality and longevity will be constant forever is an extreme one but greatly simplifies the analysis. Since a model period is twenty years and these parameters change fairly slowly this seems a reasonable assumption. An alternative extreme would be to assume that agents perfectly foresee the exact future path of parameter changes. The results of this exercise for $\sigma=3$ are shown in the Appendix. The predictions are virtually identical.
} 
Figure 8: The U.S. experience from 1800 to 1990, annual population growth rate

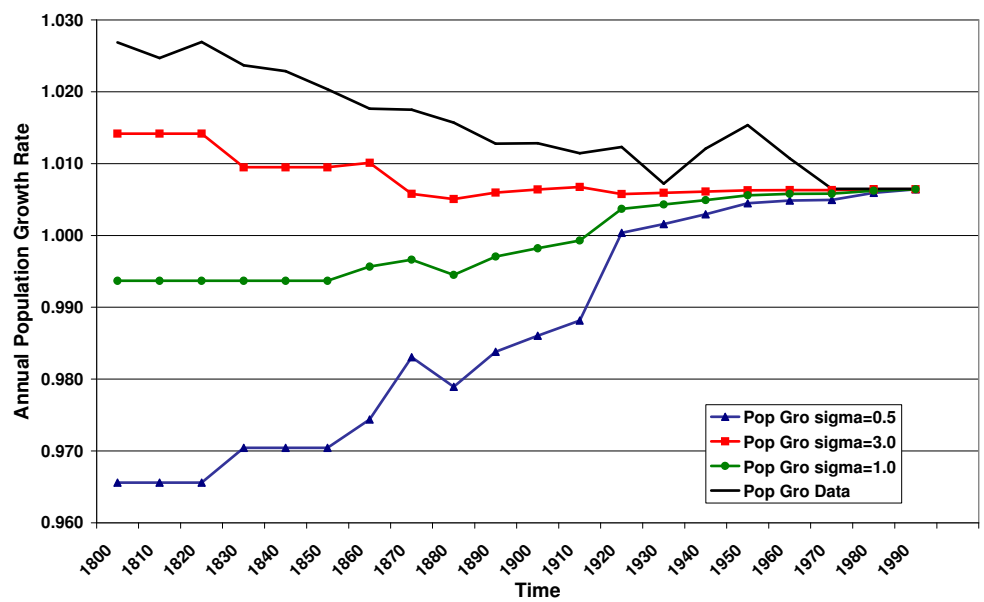

The results of this experiment are shown in Figure 7 and Figure 8. As can be seen, for high IES $(\sigma=0.5)$ the model predicts a large increase in $C B R$. The model also predicts a large increase in population growth rates in this case. These predictions are in part due to the increase in survival to adulthood and in part due to the increased growth rate in productivity, both of which always imply an increase in population growth rates for $\sigma<1$ (see Proposition 1). The first of these findings is consistent with Doepke (2005) and is the reason for his conclusion that the basic Barro-Becker model doesn't fit the facts (even though a more sophisticated model with sequential fertility choice performs slightly better).

For $\sigma=1$, the model predicts a sizable fall in $C B R$. However, since the probability of surviving to adulthood is increasing over the period, the number of surviving children $\left(C B R_{s}\right)$ may increase even if the number of births $(C B R)$ falls. In fact, if increased survival to adulthood was the only exogenous change over the period, $C B R_{s}$ (and the population growth rate, $\gamma_{N}$ ) would increase for all values of IES (see Becker and Barro (1988), Barro and Becker (1989) and Propositions 1 and 2). Indeed, the case shown with $\sigma=1$ has $C B R$ falling but $C B R_{s}$ increasing. These properties of $C B R_{s}$ are directly reflected in the predicted time path of population growth rates (see Figure 8) ${ }^{25}$ This is partly due to the fact that changes in productivity growth do not affect $C B R_{s}$ or population growth rates when $\sigma=1$ (see Propositions 1 and 2). These results are in keeping with Bar and Leukhina (2005) who find that changes in productivity have only a small effect on fertility while changes in mortality have a relatively large effect.

Finally, for low IES $(\sigma=3)$, the overall changes give rise to a predicted fall

\footnotetext{
${ }^{25}$ Comparative statics with respect to $\pi$ differ for $C B R_{s}$ and $\gamma_{N}$ but observed changes in life expectancy were small, see decomposition exercise below.
} 
in $C B R$ from 36.27 in 1800 to 17.19 in 1990. This is similar in magnitude to the reductions seen over the period in countries like the U.K. and the U.S. where CBR is estimated to have been in the range of 35 to 45 children per 1000 population in 1800 . In this case, $C B R_{s}$ is decreasing over the period, which is mainly due to increased productivity growth (see Proposition 2). In terms of population growth rates, the model predicts a fall from 1.42 to 0.65 percent per year. Hence, changes in $C B R$ predicted by the model capture about two-thirds of changes observed in the data, while model predictions of changes in population growth rates, $\gamma_{N}$, capture about one half of observed changes. ${ }^{26}$

Next, we decompose the sources of these changes (for $\sigma=3$ ) into the three components separately. That is, we ask what the model would predict for both $C B R$ and for $\gamma_{N}$ if two of the three forcing variables had stayed at their 1800 levels, while the other changed as per the experiment above. The results from these calculations are shown in Figures 9 and 10. We find that, taken one at a time, both changes productivity growth $(\gamma)$ and changes in survival probability to adulthood $\left(\pi_{s}\right)$ have sizable impacts on $C B R$. Changes in expected lifetime $(\pi)$ account for a relatively small decrease in $C B R$. As can be seen, the effect is largest for changes in $\pi_{s}$ which by itself shows a decrease in $C B R$ from 36.3 to 24.7. The effect of changing $\gamma$ alone is smaller but still significant, reducing $C B R$ from 36.3 to 28.6. The effect of a change in $\pi$ is substantially smaller, causing a reduction in $C B R$ from 36.3 to 33.0. On the other hand, the changes in both $\pi_{s}$ and $\pi$ cause population growth, $\gamma_{N}$, to increase. It is the increase in productivity growth, $\gamma$, that alleviates this so that, in sum, the effect of the three changes taken together on $\gamma_{N}$ is negative, i.e., the increase in $\gamma_{N}$ from increases in $\pi_{c y}$ and $\pi$ is more than offset by the decrease in $\gamma_{N}$ resulting from the increase in $\gamma \cdot{ }^{27}$ These finding are consistent with Mateos-Planas (2002) who focuses on population growth rates (rate of natural increase) and therefore understates the importance of mortality to understand the facts of the demographic transition.

The results of the decomposition exercise are also interesting because of their implication about the timing of fertility decline. Previous authors have criticized the hypothesis that the fertility decline was a byproduct of a reduction of infant mortality rates because of questions about the relative timing of these two changes (see for example, van de Walle (1986), Doepke (2005), Fogel (1991)). The model

\footnotetext{
${ }^{26}$ We have also experimented with even higher values for $\sigma$ (not shown here). Although the implied levels for the CBR are even higher in the earlier periods, this change is not large, and even levels of $\sigma$ close to 1,000 do not generate the entire change seen in the data.

${ }^{27}$ The range for survival probabilities conditional on reaching age $20, \pi$, are deduced from measures of expectation of life (EL) at age 20 (see Table A.4, column g). One issue related to this is that expected time in retirement has increased dramatically over the past 150 years (see Lee (2001)). We performed the same experiment using expected working life at age 20 (EWL) (i.e. the difference between expected lifetime and expected years of retirement, see Table A.4, column i ) and obtained very similar results, namely a decrease in $C B R$ from 36.95 to 19.6 and a decrease in population growth from 1.44 percent per year to 0.65 percent per year (details available upon request). The trade-off between using either one of these measures is that on the one hand, EL overstates the benefits in terms of income from having children, while EWL understates the benefits from dynasty size (since retirement is analogous to death in this case).
} 
predictions here also show that the decrease in youth mortality has very little effect before 1880. Then, from 1880 to 1950 , changes in youth mortality (from survival to age 20 of $\pi_{s}=0.64$ in 1880 to $\pi_{s}=0.96$ in 1950) account for 93 percent of the total model predicted change in CBR. However, changes in productivity growth rates (from 0 in 1800 to 1.4 percent per year in 1880) account for 90 percent of the total model predicted change in CBR for the early period.

In sum then, all three effects are quantitatively important in understanding the model predictions about the history of fertility and population growth over the last 200 years. An important requirement for the success of the model is low values for the IES in consumption.

Figure 9: Decomposition, $C B R$

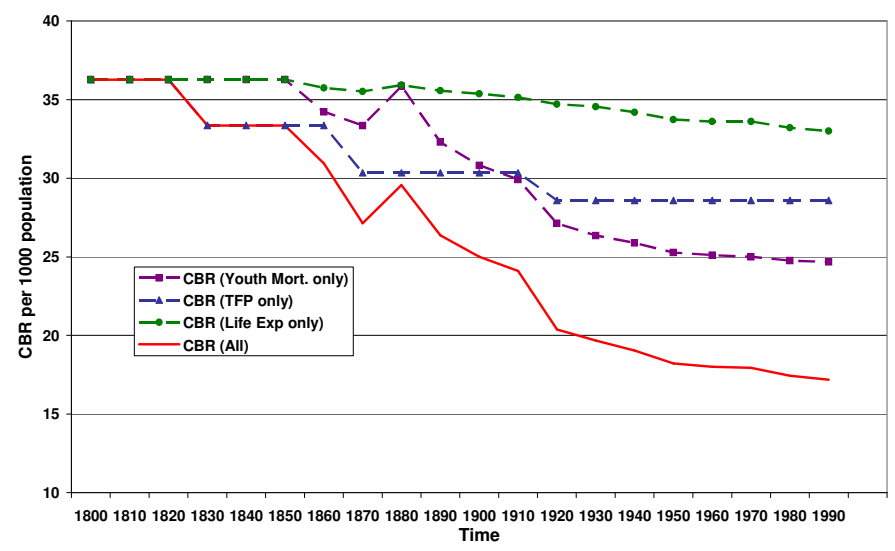

Figure 10: Decomposition, annual population growth rate

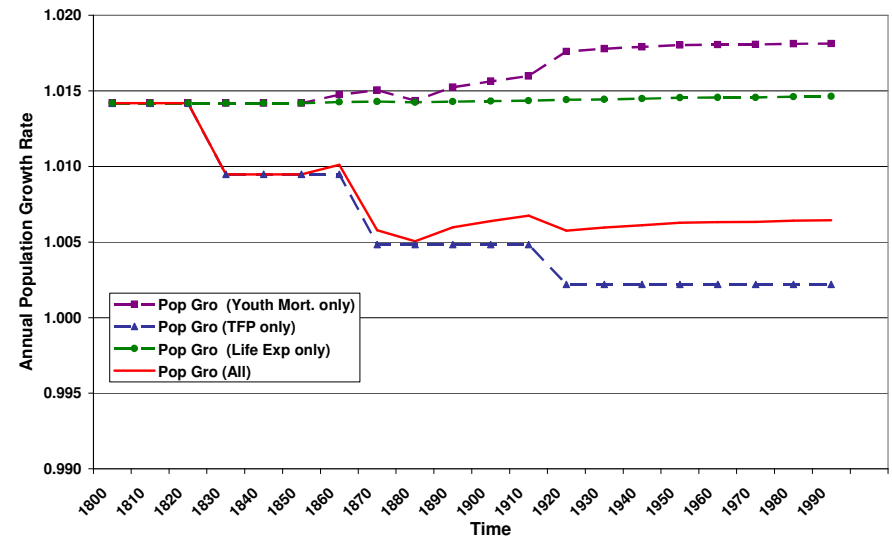




\section{Extensions}

\subsection{Lifetime, fertile period and other measures of fertility}

The discussion of the sections above shows the potential interest in studying simple Barro-Becker based models with low intertemporal substitution to understand fertility choice. One difficulty with those results is that they are stated entirely in terms of Crude Birth Rates and population growth rates, $\gamma_{N}$, across BGP's, while the usual statistics that are discussed are stated in terms of fertility rates. While there is a direct mapping between $\gamma_{N}$ and $C B R$ in the model to their data counterparts, the comparison to other measures such as Total Fertility Rate (TFR) or total Children Ever Born (CEB) to a woman (also known as Cohort Total Fertility Rate) are not so straightforward without making further assumptions about the relationship between model and data quantities. This is because we have made the simplifying assumption that all adults are identical independently of when they were born. The purpose of this assumption is to keep the number of state variables in the model at one (i.e., $N_{t}$ ) while still allowing the benefits from a birth to last for more than one model period (i.e., 20 years). One cost of this assumption is that there is no direct upper bound on the length of life, or the length of fecund life. ${ }^{28}$ This issue does not arise in a standard Barro-Becker model in which it is assumed that an adult lives for only one period. In that case, fertility of an individual over a lifetime is by assumption the same as the fertility during one period. This also results in a one state variable model. The cost however is that it is automatic that the benefits, both in terms of income and in terms of utility flows, of a child last only for one period.

The obvious remedy for this problem is to increase the number of state variables in the model and keep track of the age of the individuals in the economy. Such a model would allow us to have both realistic expected adult lifetimes and realistic fecund periods.

An alternative is to adopt the assumption that adults can only have children in the first period of their adult lives. Under the additional assumption that exactly half of these people are women there is a simple expression for the analog of CEB in the model:

$$
C E B_{\text {mod }}=\frac{N_{b t}}{\pi_{s} N_{b t-1}} \times 2=2 \times \frac{1}{\pi_{s}} \times \frac{N_{b t}}{N_{b t-1}},
$$

where $\pi_{s}$ is the probability that a birth becomes an adult.

On the BGP, $\frac{N_{b t}}{N_{b t-1}}=\gamma_{N}{ }^{29}$, and hence this can be rewritten as:

\footnotetext{
${ }^{28}$ For example, consider the Total Fertility Rate, a common measure of fertility in demography. This is defined as the total number of children a woman would have in her lifetime if she lived long enough for her fertility to be completed. If we assume that in the model, all adults are literally identical, fertility is never completed and hence, TFR from model data would be infinite by definition.

${ }^{29}$ Since $\gamma_{N}=\frac{N_{s, t}+\pi N_{t}}{N_{s, t-1}+\pi N_{t-1}}=\frac{\gamma_{N_{s}} N_{s, t-1}+\pi \gamma_{N} N_{t-1}}{N_{s, t-1}+\pi N_{t-1}}$, we must have $\gamma_{N_{s}} \equiv \frac{N_{s, t}}{N_{s, t-1}}=\gamma_{N}$. And since on a BGP, $\pi_{s, t}=\pi_{s, t-1}=\pi_{s}$ we have $\gamma_{N}=\frac{N_{b, t}}{N_{b, t-1}}$.
} 
Figure 11: Completed Fertility Model vs. Data: 1850 to 2000

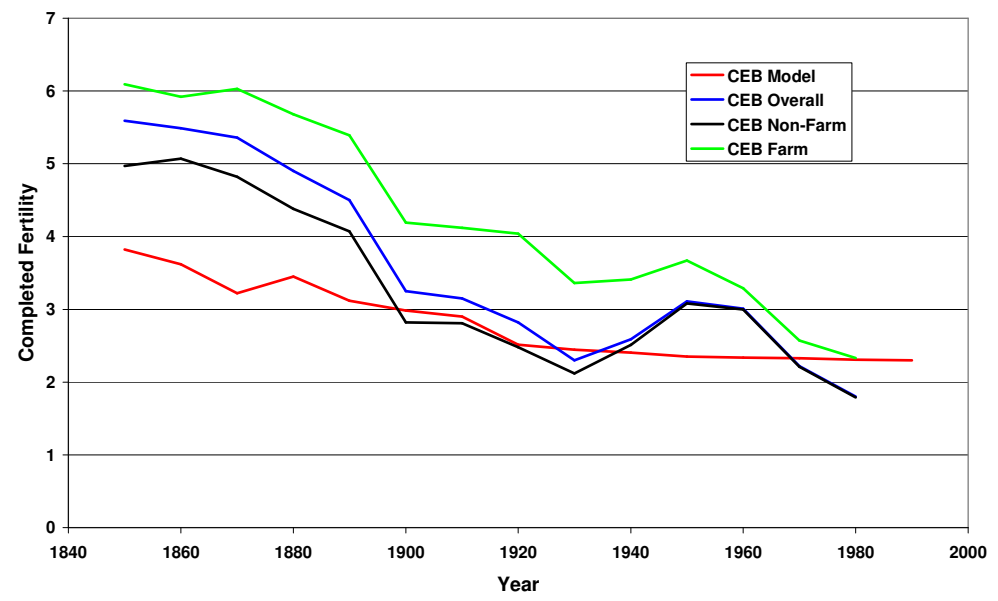

$$
C E B_{\text {mod }}=2 \times \frac{1}{\pi_{s}} \times \gamma_{N}=2 \times \frac{1}{\pi_{s}} \times \gamma_{N a n n}^{20} .
$$

Thus, for example, if $\pi_{s}=1$ and $\gamma_{N}=1, C E B=2$ - i.e., for population to be constant $\left(\gamma_{N}=1\right)$ each woman must have two children in her life.

To see how well the model tracks individual fertility we constructed the implied $C E B_{\text {mod }}$ for the simulated time series for the US from the previous section. This is shown in Figure 11 along with several cuts of the historical U.S. data on CEB over the period between 1850 and 2000. Included are the overall CEB, that for women in families living on farms, and for those not living on farms. ${ }^{30}$ As can be seen, the model predicts an increase in CEB from about 2.2 in 1990 to about 4 for women having children about 1850 . As can be seen in the figure, the increase in the data is larger, from about 2 to about 5 or 6 children, depending on which cut of the data is used. Thus, the predictions of the model capture about $2 / 3$ of the decrease in fertility for non-farm families, and only about $50 \%$ of the decline for farm families.

\subsection{Adding child quality as a choice variable}

In the model laid out so far, parents have no control over children's well being or quality, $U_{t+1}$. This simplification allowed us to derive simple intuitions and analytic comparative statics. There are various ways, however, in which parents can affect children's initial conditions. Two ways addressed frequently in the literature using dynastic models are bequests (e.g. Becker and Barro (1988), Barro and Becker (1989)) and human capital investments (e.g. Becker, Murphy and Tamura (1990), Manuelli

\footnotetext{
${ }^{30}$ This data on CEB is taken from the U.S. Census and only goes back to the 1825 to 1829 birth cohort of women. Their active fertility period began in about 1850. See Jones and Tertilt (2007) for more detail about sources.
} 
and Seshadri (2007)). In this Section, we include physical capital (i.e., bequests) into the model. As in Barro and Becker (1989), interest rates and wages are determined in equilibrium. We find that the basic intuitions go through with this change. That is, for low values of IES, population growth rates fall when productivity growth rates increase. However, the threshold for which the sign of the productivity growth effect switches is no longer $\sigma=1$. Quantitatively, effects are smaller. The intuition for this result is that when productivity growth increases exogenously, $U_{t+1}$ tends to increase, but parents now have an extra margin with which to adjust $U_{t+1}$ itself by leaving fewer bequests. This tends to dampen the complementarity / substitutability effect on fertility choice. This result suggests that even this model bypasses important effects such as increasing returns in human capital (as in Becker, Murphy and Tamura (1990), for example).

The representative dynasty problem we are interested in is given by

$$
\operatorname{Max}_{\left\{C_{t}, N_{t}, K_{t}\right\}} \quad U_{0}\left(\left\{C_{t}, N_{t}, K_{t}\right\}\right)=\sum_{t=0}^{\infty} \beta^{t} N_{t}^{\eta+\sigma-1} \frac{C_{t}^{1-\sigma}}{1-\sigma}
$$

s.t.

$$
\begin{aligned}
& C_{t}+\theta_{s t} N_{s t}+X_{t} \leq w_{t} N_{t}+r_{t} K_{t} \\
& K_{t+1} \leq(1-\delta) K_{t}+X_{t}, \\
& N_{t+1} \leq \pi N_{t}+N_{s t} \\
& \left(N_{0}, K_{0}\right) \text { given. }{ }^{31}
\end{aligned}
$$

The first order condition with respect to $K_{t+1}$ and $N_{t+1}$ together with the budget constraint, boil down to the following system of equations governing the solution to this (partial equilibrium) problem:

$$
\begin{aligned}
& \gamma_{c t}^{\sigma} \gamma_{N t}^{1-\eta}=\beta\left(r_{t+1}+1-\delta\right) \\
& \theta_{t}\left(r_{t+1}+1-\delta\right)=\left[\frac{(\eta+\sigma-1)}{(1-\sigma)} \frac{C_{t+1}}{N_{t+1}}+\left[w_{t+1}+\theta_{t+1} \pi\right]\right] \\
& \frac{C_{t}}{N_{t}}+\theta_{t} \gamma_{N t}+\frac{K_{t+1}}{N_{t+1}} \gamma_{N t}=\left[w_{t}+\pi \theta_{t}\right]+\left(r_{t}+1-\delta\right) \frac{K_{t}}{N_{t}}
\end{aligned}
$$

To close the model, wages and interest rates are determined in equilibrium by a firm hiring labor and capital to maximize profits with a CRS production function, $F\left(K_{t}, \gamma^{t} N_{t}\right)=A K_{t}^{\alpha}\left(\gamma^{t} N_{t}\right)^{1-\alpha}$. That is,

$$
\begin{aligned}
& r_{t}=F_{K}\left(K_{t}, \gamma^{t} N_{t}\right) \\
& w_{t}=F_{N}\left(K_{t}, \gamma^{t} N_{t}\right)
\end{aligned}
$$

On a balanced growth path, we have $\gamma_{c, t}=\gamma_{c}=\gamma, \gamma_{N, t}=\gamma_{N}, \gamma_{C}=\gamma_{K}=\gamma \gamma_{N}$, wages grow at $\gamma$ and interest rates are constant. Denoting detrended variables by $\widehat{x}$ the above equations become:

$$
\gamma_{N}=\frac{[\beta(r+1-\delta)]^{\frac{1}{1-\eta}}}{\gamma^{\frac{\sigma}{1-\eta}}}
$$

${ }^{31}$ Notice that since as before one implicit assumption is that all alive adults are identical in this model, we have to assume that parents share their assets equally among all surviving family members (including themselves), so that initial conditions of all households are the same. 


$$
\begin{aligned}
& \widehat{c}^{*}=\left[\widehat{w}+\pi \theta_{s}\right]+\gamma(r+1-\delta) \widehat{k}^{*}-\theta_{s} \gamma_{N}-\widehat{k}^{*} \gamma_{N} \gamma \\
& r+1-\delta=\frac{\gamma}{\theta_{s}} \frac{(\eta+\sigma-1)}{(1-\sigma)} \widehat{c}^{*}+\frac{\gamma}{\theta_{s}} \widehat{w}+\pi \gamma \\
& r=\alpha A \widehat{k}^{* \alpha-1} \\
& \widehat{w}=(1-\alpha) A \widehat{k}^{* \alpha}
\end{aligned}
$$

From the first equation above, it is clear that - as usual in Barro-Becker type models, higher interest rates are typically associated with higher population growth. In light of the comparative statics results above, suppose the rate of productivity growth, $\gamma$, increases. Holding the interest rate fixed (i.e., in partial equilibrium), this decreases the population growth rate. However, the interest rate is endogenous. In response to an increase in productivity growth the BGP level of the capital-people ratio, $\widehat{k}^{*}$, decreases and hence the interest rate increases which tends to increase population growth. Whether the population growth increases or decreases depends on all parameters, in particular the side of the parameter space one chooses:

I. In the case where $0<\eta<1$ and $0<\sigma<1$ the positive effect from the interest rate is large since $\frac{1}{1-\eta}>1$. However, since in this case, we also have $\frac{\sigma}{1-\eta} \geq 1$, the direct negative effect from an increase in productivity growth is also large.

II. In the case where $\sigma>1$ and $\eta \leq 1-\sigma<0$, the positive effect from the interest rate is small since $\frac{1}{1-\eta}<1$. However, since in this case, we also have $\frac{\sigma}{1-\eta} \leq 1$, the direct negative effect from an increase in productivity growth is also small.

In the case where $\eta=1-\sigma$ a sufficient condition for the population growth rate to be decreasing in productivity growth is $\sigma>1-\alpha$ (this condition is necessary and sufficient in the case where there is full depreciation in physical capital and people live for only one period, i.e. $1-\delta=\gamma \pi=0$, the analogue without capital was $\sigma>1$ ). Since $\alpha>0$, it is possible that the net effect on population growth from an increase in productivity is negative even if $\sigma<1$. Nevertheless, the typical values used in the fertility literature $(\sigma \approx 0.5)$ with a capital share of $\alpha \approx 0.4$ do not satisfy this condition.

Quantitatively, experiments analogous to those in Section 6 (calibrating to 1990 and changing youth mortality, longevity and productivity growth as observed in the US since 1800), give results that are quite similar. Using the same parameters as before together with $\sigma=3, \alpha=0.4$ and capital depreciation of $5 \%$ per annum, the model predicts a fall in CBR from 30.2 to 17.19 (compared to 36.3 to 17.19 in the model without capital), while for $\sigma=0.5 \mathrm{CBR}$ falls from only 20.3 to 17.19 (compared to an increase from 2 to 17.19 in the model without capital). For population growth rates, however, the effect of increased productivity which decreases population growth no longer dominates the effect from mortality and longevity which increases population growth rates. This produces a slight overall increase from $0.47 \%$ per annum to $0.65 \%$ per annum when $\sigma=3$ and a large increase from $-0.01 \%$ to $0.65 \%$ per annum when $\sigma=0.5$. 
Hence, the choice of $\sigma$ is still crucial for qualitative and quantitative predictions of dynastic models even if parents have some control over children's initial conditions (through bequests in this case) and hence, their well-being, $U_{t+1}$.

\subsection{Other Extensions}

There are many important extensions that could be done to give a clearer picture of the quantitative properties of models like this. These include, but are not limited to:

1. Education as Child Quality

Many of the authors who have worked on the history of fertility have emphasized the role of changes in education over the last 200 years. Examples include Becker, Murphy and Tamura (1990), Galor and Weil (2000), FernandezVillaverde (2001), Soares (2003), Doepke (2004) and (2005), Manuelli and Seshadri (2006), Rosenzweig (1990) and many others. From a formal point of view, this is much like including a capital stock as discussed above, i.e., it is another way for parents to affect the level of utility of their children. The technology and policy issues that arise might be quite different however. Examples of the latter include the kinds of regulatory changes that have taken place, e.g., compulsary schooling, see Doepke (2005) for example.

2. Changes in costs of children

Throughout, we have assumed that the costs of raising a child to adulthood have been unchanged over the period. However, when one adopts a broad view of what determines these costs - e.g., subtracting out any direct input from the child on a farm - this is clearly a strong assumption. Indeed, the relative availability of land in the U.S. and the resulting implications for the size of net costs of children may be one of key reasons the model predicts fertility that is too low for the early years (and also one of the reasons that fertility was so much higher in the U.S. than it was in the U.K.). Clearly the analysis would benefit greatly from a more careful accounting of the costs of children along these dimensions.

\section{Endogenous Mortality}

In our analysis, we have assumed that survival probabilities are exogenous to the decision maker. While this is probably a reasonable assumption about many of the improvements in health over the period (the development of the germ theory of disease, the advent of pasteurization of milk and vaccinations), there are also many aspects of health that affect these probabilities are in fact chosen. Indeed, some authors have explicitly modeled this choice (e.g., Fernandez-Villaverde (2001) and Kalemli-Ozcan (2002) and (2003)). Extensions along this line would also be fruitful. 


\section{Conclusion}

In models of fertility choice based on parental altruism à la Barro and Becker, we have shown that a key model feature is the decision on whether the number of children and utility of those children are complements or substitutes. This is important both qualitatively and quantitatively. When family size and utility of offspring are complements, as is commonly assumed, there is a strong force to increase fertility when conditions improve, either over time, or across countries. This is counter to what is seen in the data. On the other hand, when they are substitutes, as we have shown here, there is a strong force in the opposite direction. Further, we have shown that this choice is closely related to the choice of the intertemporal elasticity of substitution in consumption which through the desire to smooth consumption also affects the size of the fertility response to changes in mortality, longevity and productivity growth.

The effects laid out in this paper are purely preference driven and hence present in many extensions including formulations with the possibility of leaving bequests or educating children. Hence, the results in this paper shed new light on previous work using Barro-Becker type preferences and are qualitatively relevant for any question being addressed using these preferences.

As we have seen, the ability of the model quantities to match key features of the data also depends critically on the choice of the IES. These quantitative results

help reconcile seemingly contradictory results in the quantitative literature addressing trends in fertility.

\section{References}

Ales, L. and P. Maziero, (2007), "Accounting for Private Information," working paper.

Alvarez, F., (1999), "Social Mobility: The Barro-Becker Children Meet the LaitnerLoury Dynasties," Review of Economic Dynamics, 2(1): 65-103.

Bar, M. and Leukhina, O., (2005), "A Model of Historical Evolution of Output and Population," working paper, University of North Carolina.

Barro R. J., and Becker, G. S., (1989), "Fertility Choice in a Model of Economic Growth," Econometrica, 57, (2): 481-501.

Becker, G. S. and Barro, R. J., (1988), "A Reformulation of the Theory of Fertility," Quarterly Journal of Economics, 103: 1-25.

Becker G. S., Murphy K. M., and Tamura, R., (1990), "Human Capital, Fertility, and Economic Growth," Journal of Political Economy, 98:12-37.

Benhabib, J., and K. Nishimura, (1993), "Endogenous Fertility and Growth," in General Equilibrium, Growth and Trade II, ed. by R.Becker, M.Boldrin, R.Jones and W.Thomson, Academic Press, San Diego, 237-247.

Ben-Porath, Y., (1976), "Fertility Response to Child Mortality: Micro Data from Israel," Journal of Political Economy, 84 (4): S163-S178. 
Blanchard, O.J. (1985) "Debt, Deficits and Finite Horizons", Journal of Political Economy 93, 223-247

Boldrin, M. and Jones, L. E., (2002), "Mortality, Fertility, and Saving in a Malthusian Economy," Review of Economic Dynamics, 5 (4): 775-814.

Boldrin, M., DeNardi, M. and Jones, L. E., (2005), "Fertility and Social Security," working paper, University of Minnesota.

Clark, Gregory, "The Secret History of the Industrial Revolution," Working paper, UC Davis, 2001

De la Croix, D. and Doepke, M., (2003), "Inequality and Growth: Why Differential Fertility Matters," American Economic Review, 93, (4): 1091-1113.

Department of Commerce, Bureau of the Census, (1910), "U.S. Life Tables 1890, 1901, 1910 and 1901 to $1910 . "$

Doepke, M., (2004), "Accounting for Fertility Decline During the Transition to Growth," Journal of Economic Growth, 9(3): 347-383.

Doepke, M., (2005), "Child Mortality and Fertility Decline: Does the BarroBecker Model Fit the Facts?," Journal of Population Economics, 18: 337-366.

Eckstein, Z., Mira, P. and Wolpin, K. I., (1999), "A Quantitative Analysis of Swedish Fertility Dynamics: 1751-1990," Review of Economic Dynamics, 2:137-165.

Ehrlich, I. and F. T. Lui (1991), "Intergenerational Trade, Longevity, and Economic Growth," Journal of Political Economy, Vol. 99, No. 5 (Oct.), pp. 1029-1059

Fernandez-Villaverde, J., (2001), "Was Malthus Right? Economic Growth and Population Dynamics," Working paper, University of Pennsylvania.

Fogel R. W., (1990). "The Conquest of High Mortality and Hunger in Europe and America: Timing and Mechanisms," NBER Historical Working Papers 0016, National Bureau of Economic Research, Inc.

Galor, O. and Weil, D. N., (2000), "Population, Technology, and Growth: From Malthusian Stagnation to the Demographic Transition and Beyond," American Economic Review, 90 (4): 806-28.

Greenwood, J. and Seshadri, A., (2002), "The U.S. Demographic Transition," American Economic Review Papers and Proceedings, 92 (2): 153-159.

Greenwood, J. and G. Vandenbroucke, (2005), "Hours Worked: Long Run Trends," WP \# 11629, NBER, September 2005.

Hacker, J. David, (2003), "Rethinking the "Early" Decline of Marital Fertility in the United States," Demography, Vol. 40, No. 4., pp. 605-620.

Haines, M. R., (1994a), "Estimated Life Tables for the United States, 1850-1900," NBER Historical Paper \#59.

Haines, M. R., (1994b), "The Population of the United States, 1790-1920", NBER Historical Working Paper No. 56.

Human Mortality Database. University of California, Berkeley (USA), and Max Planck Institute for Demographic Research (Germany). Available at www.mortality.org or www.humanmortality.de 
Jones, L.E., and A. Schoonbroodt, (2006), "Baby Busts and Baby Booms: The fertility Response to Shocks in Dynastic Models", working paper.

Jones, L.E., and M. Tertilt, (2007), "An Economic History of Fertility in the U.S.: 1826-1960," working paper.

Kalemli-Ozcan, S. (2002), "Does the Mortality Decline Promote Economic Growth?," Journal of Economic Growth, 7 (4): 411-39.

Kalemli-Ozcan, S., (2003), "A Stochastic Model of Mortality, Fertility, and Human Capital Investment," Journal of Development Economics, 70 (1): 103-18.

Lee, C., (2001) "The expected length of male retirement in the United States, 1850-1990," Journal of Population Economics 14: 641-650

Maddison, Angus, Monitoring the World Economy: 1820/1992, OECD Development Center, 1995

Manuelli, R. E. and Seshadri, A., (2006), "The Contribution of Human Capital to Development," working paper, University of Wisconsin.

Mateos-Planas, X., (2002), "The Demographic Transition in Europe: A Neoclassical Dynastic Approach," Review of Economic Dynamics, 5: 646-680.

Mitchell, Brian R. International Historical Statistics: Europe, 1750-1993. New York, N.Y.: Stockton Press, 1998.

National Center for Health Statistics, (1998), "Hist 290: Death Rates for Selected Causes by 10 year Age Groups, Race and Sex: Death Registration States, 1900 to 1932 and U.S., 1933 to 1998" from "Historical Vital Statistics of the United States: 1890 to 1998." Available at: http://www.cdc.gov/nchs/datawh/statab/unpubd/mortabs/hist290.htm.

Rosenzweig, M. R., (1990), "Population Growth and Human Capital Investments: Theory and Evidence," Journal of Political Economy 98, S38-S70.

Sah, R. K., (1991), The Effects of Child Mortality Changes on Fertility Choice and Parental Welfare, Journal of Political Economy, 99, (3): 582-606.

Soares, R. R., (2003), "Mortality Reductions, Educational Attainment, and Fertility Choice," Working paper, University of Maryland.

Topkis, Donald (1998), Supermodularity and Complementarity, Princeton University Press, Frontiers of Economic Research Series.

van de Walle, F., (1986), "Infant Mortality and the European Demographic Transition," Chapter 4 of The Decline of Fertility in Europe: The Revised Proceedings of a Conference on the Princeton European Fertility Project, edited by Coale AJ, Cotts Watkins S (ed) 201-233. Princeton: Princeton University Press.

Wolpin, K.I., (1997), "Determinants and Consequences of the Mortality and Health of Infants and Children," Chapter 10 of Handbook of Population and Family Economics Vol. IA, edited by Rosenzweig M. R., and O. Stark, Eds., 483-557. Amsterdam: Elsevier.

Wrigley, E.A., Davies, R.S., Oeppen, J.E., and R.S. Schofield, English Population History from Family Reconstitution 1580-1837, Cambridge University Press, Cambridge, 1997 


\section{A Appendix}

\section{A.1 Remarks and issues regarding the dynasty utility}

This formulation for the dynasty utility flow gives rise to some very useful simplifications that we will exploit below. One disadvantage of it however, is that it is not equivalent to logarithmic utility when $\sigma=1$. However, when $\eta=1-\sigma$ and $\sigma \rightarrow 1$, the preferences, will converge to those given by the utility function $\sum \beta^{t} \log \left(C_{t}\right)$. See Bar and Leukhina (2006) for an explicit derivation of Barro-Becker preferences with an IES equal to one.

Another concern may be the fact that, for $\sigma>1, U_{0}=-\infty$ if for any $i$ and $t$, $n_{i t}=0$. From the dynastic point of view, if at any point in time, any descendant has zero children that branch of the dynasty has $-\infty$ continuation utility. When $0 \leq \sigma<1$, this is not an issue however - the dynasty just gets $U=0$. This is particularly relevant for questions such as those in Doepke (2005) and Kalemli-Ozcan (2003) where the probability to end up with zero children is positive. One can get around this problem by adding small constants to consumption and children in the utility and thereby preserve the properties of the present model to a large extent while bounding utility away from $-\infty .^{32}$

Finally, there are ways to combine cases of low (high) IES and complementarity (substitutability). For example, write the period utility in the dynasty problem as

$$
\frac{\left[\mu N_{t}^{\rho}+(1-\mu) C_{t}^{\rho}\right]^{\frac{1-\psi}{\rho}}}{1-\psi}
$$

Then, as long as $\rho<1-\psi, C$ and $N$ are complements in utility and substitutes if $\rho>1-\psi$. This implies that to overturn the effect of the IES, population and consumption have to be very complementary/substitutable. The disadvantage of such a formulation is that the basic interpretation of $g$ and $U_{t+1}$ are no longer straightforward and disaggregation becomes difficult. The formulation above, implicitly assumes $\rho=0, \mu(1-\psi)=\eta+\sigma-1$ and $(1-\mu)(1-\psi)=1-\sigma$.

\footnotetext{
${ }^{32}$ Details available upon request.
} 


\section{A.2 Results from Section 5}

Table A.1: Changing productivity growth

\begin{tabular}{c|cc|cc|cc}
\hline \hline $\begin{array}{c}\text { Productivity Growth } \\
\gamma_{\text {ann }}\end{array}$ & \multicolumn{2}{|c|}{$\sigma=0.5$} & \multicolumn{2}{c|}{$\sigma=1.0$} & \multicolumn{2}{c}{$\sigma=3.0$} \\
& $\gamma_{N, \text { ann }}$ & $C B R_{s, \text { ann }}$ & $\gamma_{N, \text { ann }}$ & $C B R_{s, \text { ann }}$ & $\gamma_{N, \text { ann }}$ & $C B R_{s, \text { ann }}$ \\
\hline 1.00 & 0.98 & 5.25 & 1.00 & 15.38 & 1.013 & 21.37 \\
1.01 & 0.99 & 10.49 & 1.00 & 15.38 & 1.007 & 18.45 \\
1.02 & 1.00 & 15.38 & 1.00 & 15.38 & 1.00 & 15.38 \\
\hline \hline
\end{tabular}

Table A.2: Changing survival to adulthood (STA)

\begin{tabular}{c|cc|ccc}
\hline \hline & $\pi_{c y}$ & $\theta_{s} / w$ & $\gamma_{N, \text { ann }}$ & $C B R_{\text {ann }}$ & $C B R_{s, a n n}$ \\
& $(\mathrm{STA})$ & & & & \\
\hline \multirow{5}{*}{$\sigma=0.5$} & 0.6 & 0.85 & 0.993 & 19.68 & 11.81 \\
& 0.8 & 0.80 & 0.997 & 17.51 & 14.01 \\
& 1.0 & 0.77 & 1.00 & 15.38 & 15.38 \\
\hline \multirow{5}{*}{$\sigma=1.0$} & 0.6 & 0.65 & 0.996 & 22.46 & 13.48 \\
& 0.8 & 0.61 & 0.998 & 18.31 & 14.65 \\
& 1.0 & 0.59 & 1.00 & 15.38 & 15.38 \\
\hline \multirow{3}{*}{$\sigma=3.0$} & 0.6 & 0.26 & 0.998 & 24.16 & 14.50 \\
& 0.8 & 0.24 & 0.999 & 18.80 & 15.04 \\
& 1.0 & 0.23 & 1.00 & 15.38 & 15.38 \\
\hline \hline
\end{tabular}


Table A.3: Changing expected lifetime (EL)

\begin{tabular}{c|cc|cc}
\hline \hline & $\pi_{a n n}$ & $\frac{T}{1-\pi}$ & $\gamma_{N, a n n}$ & $C B R_{s}$ \\
\hline \multirow{5}{*}{$\sigma=0.5$} & 0.923 & 25 & 0.979 & 15.60 \\
& 0.959 & 35 & 0.993 & 15.26 \\
& 0.971 & 45 & 1.00 & 15.38 \\
\hline \multirow{5}{*}{$\sigma=1.0$} & 0.923 & 25 & 0.991 & 19.56 \\
& 0.959 & 35 & 0.997 & 17.00 \\
& 0.971 & 45 & 1.00 & 15.38 \\
& & & & \\
\hline & 0.923 & 25 & 0.998 & 21.84 \\
$\sigma=3.0$ & 0.959 & 35 & 0.999 & 18.01 \\
& 0.971 & 45 & 1.00 & 15.38 \\
& & & & \\
\hline \hline
\end{tabular}

\section{A.3 Time series used in Section 6}

Data Sources for Table A.4:

- Column b, $\gamma$ (annual productivity growth rate): is from the data on real wages in Greenwood and Vanderbroucke (2005), from 1830 to 1988, for the period from 1800 to 1830, we assumed $\gamma=1.00$;

- Column c, $\pi_{i}$ (survival probability from age 0 to age 1 ): is derived from data on Infant Mortality Rates, IMR's from:

1850 to 1900 are from Haines (1994a), U.S. Model, Total Population Both Sexes; 1800 to 1840 are assumed to be the same as 1850;

1910 is taken from U.S. Department of Commerce (1910);

1920 to 1990 are from National Center for Health Statistics (1998);

- Column d, $\pi_{i c}$ (survival probability to age 5, conditional on surviving to age 1): 1850 to 1900 are derived from year by year death rates in Haines (1994a), U.S.

Model, Total Population Both Sexes;

1800 to 1840 are assumed to be the same as 1850;

1910 is derived from year by year death rates from U.S. Department of Commerce (1910);

1920 to 1990 are from National Center for Health Statistics (1998);

- Column e, $\pi_{c y}$ (survival probability to age 20,conditional on surviving to age 5): 1850 to 1900 are derived from year by year death rates in Haines (1994a), U.S. Model, Total Population Both Sexes; 
Table A.4: Annual Data Used for the Time Series Experiment in Section 6, U.S.

\begin{tabular}{|c|c|c|c|c|c|c|c|c|c|c|}
\hline $\begin{array}{c}\mathrm{a} \\
\text { Year }\end{array}$ & $\begin{array}{l}\mathrm{b} \\
\gamma\end{array}$ & $\begin{array}{c}\mathrm{c} \\
\pi_{i}\end{array}$ & $\begin{array}{c}\mathrm{d} \\
\pi_{i c}\end{array}$ & $\begin{array}{c}\mathrm{e} \\
\pi_{c y}\end{array}$ & $\begin{array}{c}\mathrm{f} \\
\frac{T}{1-\pi} \\
\mathrm{EL}\end{array}$ & $\begin{array}{c}\mathrm{g} \\
\pi_{a n n} \\
\mathrm{EL}\end{array}$ & $\begin{array}{c}\frac{\mathrm{h}}{T} \\
\frac{T}{1-\pi} \\
\text { EWL }\end{array}$ & $\begin{array}{c}\mathrm{i} \\
\pi_{a n n} \\
\mathrm{EWL}\end{array}$ & $\underset{C B R}{\stackrel{\mathrm{j}}{\text { data }}}$ & $\begin{array}{c}\mathrm{k} \\
P G \\
\text { data }\end{array}$ \\
\hline 1800 & 1.000 & 0.771 & 0.895 & 0.916 & 38.41 & 0.964 & 36.73 & 0.961 & 45.40 & 1.027 \\
\hline 1810 & 1.000 & 0.771 & 0.895 & 916 & 38.41 & 0.964 & 36.73 & 0.961 & 45.82 & 1.025 \\
\hline 1820 & 1.000 & 0.771 & 0.895 & 0.916 & 38.41 & 0.964 & 36.73 & 0.961 & 45.12 & 1.027 \\
\hline 1830 & 1.007 & 0.771 & 0.895 & 0.916 & 38.41 & 0.964 & 36.73 & 0.961 & 45.18 & 1.024 \\
\hline 1840 & 1.007 & 0.771 & & 0.916 & 38.41 & 0.964 & 36.73 & 0.961 & 48.17 & 1.023 \\
\hline 1850 & 007 & 0.771 & 0.895 & 916 & 38.41 & 0.964 & 36.73 & 0.961 & 46.05 & .020 \\
\hline 1860 & 1.007 & 0.803 & 0.909 & 0.926 & 40.32 & 0.966 & 38.69 & 0.964 & 45.19 & 1.018 \\
\hline 1870 & 1.014 & 0.816 & 0.917 & 0.933 & 41.14 & 0.967 & 40.31 & 0.966 & 39.30 & 1.017 \\
\hline 1880 & 1.014 & 0.775 & 0.899 & 0.920 & 39.65 & 0.966 & 38.03 & 0.963 & 35.16 & 1.016 \\
\hline 1890 & 1.014 & 0.840 & 0.921 & 0.934 & 40.96 & 0.967 & 38.70 & 0.964 & 32.71 & 1.013 \\
\hline 1900 & 1.014 & 0. & 0.930 & 0.940 & 41.73 & 0.968 & 38.80 & 0.964 & 28.58 & 1.013 \\
\hline 191 & & 0.88 & $0.9^{2}$ & & 42. & 0.9 & 39 & & 27.50 & 1.011 \\
\hline 1920 & 1 & 0.908 & 0.990 & 0.992 & 44.45 & 0.971 & 40.60 & 0.967 & 24.92 & 1.012 \\
\hline & 1 & 0.931 & 0.994 & 0.9 & 45.14 & 0.971 & 40.19 & 0.966 & 19.05 & 1.007 \\
\hline 19 & & 0.945 & 0.997 & 0.99 & 46.77 & 0.973 & 41.01 & 0.967 & 18.60 & 1.012 \\
\hline 1950 & 1.018 & 96 & 0.998 & 0.998 & 49 & 0.9 & 42.33 & 0.9 & 23.00 & 1.015 \\
\hline 1960 & 1.018 & 0.973 & 0.999 & 0.998 & 49.65 & 0.975 & 41.77 & 0.968 & 22.70 & 1.011 \\
\hline 197 & 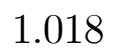 & .977 & 0.9 & 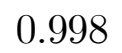 & 49.63 & 0.975 & 41.04 & 0.967 & 17.40 & 1.006 \\
\hline 198 & 1. & 0.98 & 0.999 & 0 & 51.73 & 0.9 & 40.46 & 0.966 & 15.10 & 1.006 \\
\hline 1990 & 1.018 & 0.990 & 1.000 & 0.999 & 52.95 & 0.977 & 40.29 & 0.966 & 15.80 & 1.006 \\
\hline
\end{tabular}

1800 to 1840 are assumed to be the same as 1850 ;

1910 is derived from year by year death rates from U.S. Department of Commerce (1910);

1920 to 1990 are from National Center for Health Statistics (1998);

- Column f, $\frac{T}{1-\pi}$ (EL) (expectation of life at age 20): is taken from Lee (2001), Column B;

- Column g, $\pi_{a n n}$ (EL) (annual adult survival rate): is derived from Column f;

- Column h, $\frac{T}{1-\pi}$ (EWL) (expected working life at age 20): is taken from Lee (2001), Column C;

- Column i, $\pi_{a n n}$ (EWL) (annual adult survival rate in the workforce): is derived from Column h;

- Column j, $C B R$ (crude birth rate, annual): 
1800 to 1930 are taken from Hacker (2003), Figure 1, 1940 to 1990 are from Haines (1994b), Table 3;

- Column k, PG (population growth rate, annual): are taken from Haines, (1994b), Table $1, \mathrm{RNI}=$ rate of natural increase (net of immigration).

\section{A.4 The case of the U.K. ${ }^{33}$}

In this Section, we perform the same experiment as in Section 6 but using data for the United Kingdom (England and Wales for the most part). The results are quite similar: we capture about two-thirds of the change in CBR and one half of the change in population growth.

The fertility experience in the U.K. over the past 200 years is similar to that of the U.S., except that levels in 1800 were lower already. because of this the decrease, both in CBR and population growth, was smaller. Mortality was also lower in the U.K. than it was in the U.S. around that time. Finally, our estimates of productivity growth suggest the latter was higher in 1800 as well. Since, fertility, mortality and productivity growth are very similar in the two countries in 1990, all these observations are consistent with our theory and one would expect the model to capture the same fraction of changes in fertility and population growth.

Data Sources for Table A.5:

- Column b, $\gamma$ (annual productivity growth rate) from annual growth rate in GDP per capita, (log GDP HP filtered $\lambda=400)$ :

1800 to 1865 from Clark (2001),

1850 to 1990 from Maddison (1995), p. 194, rescaled to match Clark in 1850;

- Column c,d,e, $\left(\pi_{i}, \pi_{i c}, \pi_{c y}\right)$ (survival rates from age specific mortality rates) 1800 to 1837 from Wrigley et al. (1997), Table 6.1, p.215, 1841 to 1990 from Human Mortality Database;

- Column f, $\frac{T}{1-\pi}$ (EL) (expectation of life at age 20): 1841 to 1990 from Human Mortality Database, 1800 to 1836 set constant at 39 years;

- Column g, $\pi_{a n n}$ (EL) (annual adult survival rate): derived from Column f;

- Column h, $C B R$ (crude birth rate, annual): 1800 to 1871 from Wrigley et al. (1997), 1871 to 1986 from Mitchell (1998)

- Column i, $C B R$ HP filtered (crude birth rate, annual): Column h HP filtered, $\lambda=400$;

- Column j, PG (population growth rate, annual): 1800 to 1837 from Wrigley et al. (1997), Table 6.1, p.215 1841 to 1990 from Human Mortality Database;

- Column k, PG HP filtered (population growth rate, annual): Column j HP filtered, $\lambda=400$.

\footnotetext{
${ }^{33}$ We thank Micheal Bar and Oksana Leukhina for help with data sources,
} 
Figure A.1: The U.K. experience from 1800 to 1990, $C B R$

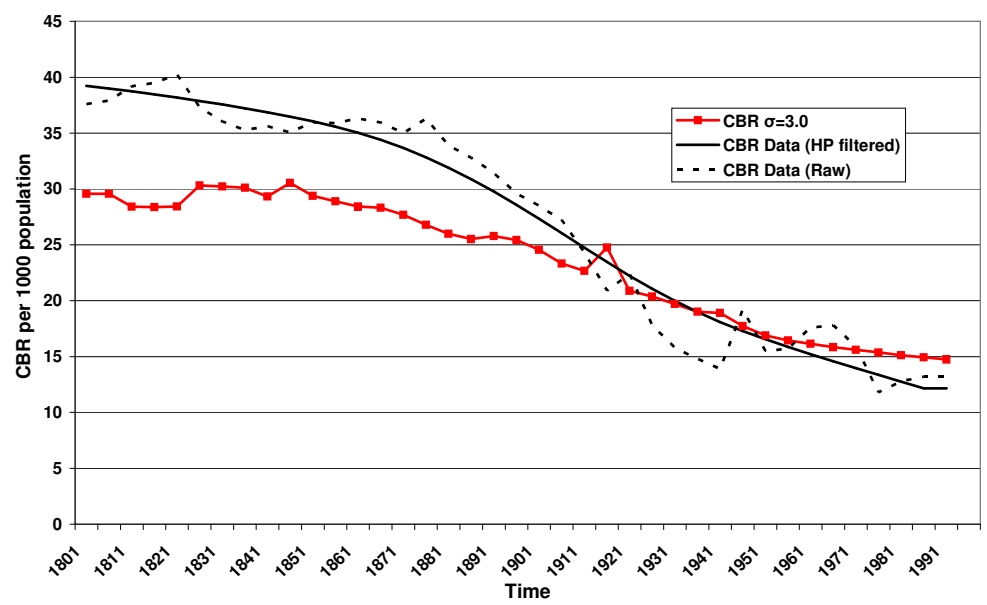

Figure A.2: The U.K. experience from 1800 to 1990, annual population growth rate

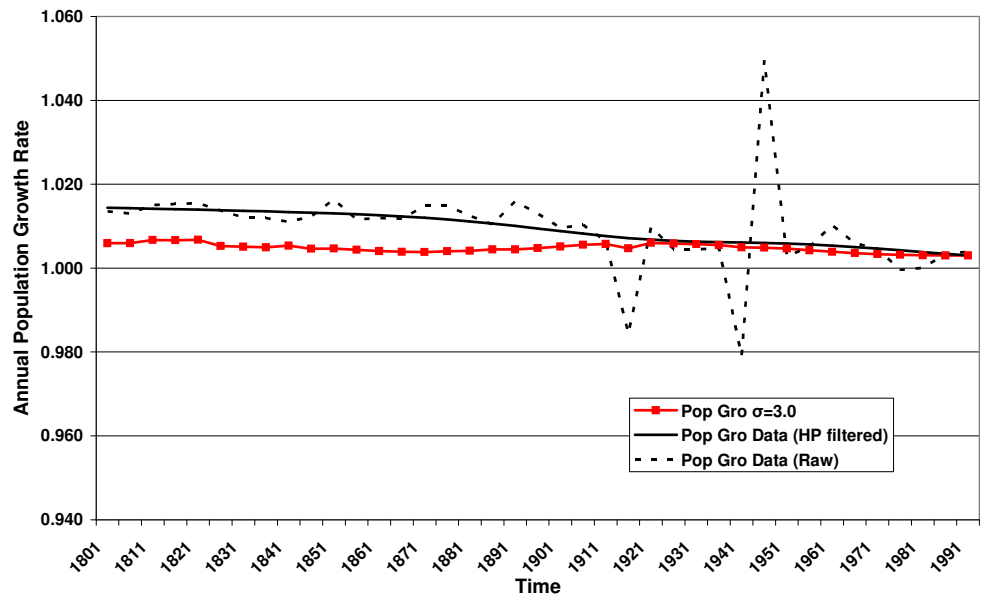


Table A.5: Annual Data Used for the Time Series Experiment in Section A.4, U.K.

\begin{tabular}{|c|c|c|c|c|c|c|c|c|c|c|}
\hline $\begin{array}{c}\mathrm{a} \\
\text { Year }\end{array}$ & $\begin{array}{l}\mathrm{b} \\
\gamma\end{array}$ & $\begin{array}{c}\mathrm{c} \\
\pi_{i}\end{array}$ & $\begin{array}{c}\mathrm{d} \\
\pi_{i c}\end{array}$ & $\begin{array}{c}\mathrm{e} \\
\pi_{c y}\end{array}$ & $\begin{array}{c}\mathrm{f} \\
\frac{T}{1-\pi} \\
\mathrm{EL}\end{array}$ & $\begin{array}{c}\mathrm{g} \\
\pi_{a n n} \\
\mathrm{EL}\end{array}$ & $\begin{array}{c}\mathrm{h} \\
C B R \\
\text { data }\end{array}$ & $\begin{array}{c}\mathrm{i} \\
C B R \\
\text { нР }\end{array}$ & $\begin{array}{c}\mathrm{j} \\
P G \\
\text { data }\end{array}$ & $\begin{array}{c}\mathrm{k} \\
P G \\
\text { нР }\end{array}$ \\
\hline 1801 & 06 & 863 & 0.884 & 864 & 39.00 & 0.965 & 37.60 & 39.23 & 1.014 & .014 \\
\hline 1806 & & 862 & & & 39.00 & 0965 & 37.90 & & 1.013 & .014 \\
\hline 1811 & 006 & 867 & 870 & 925 & 39.00 & 0.965 & 39.18 & 38.72 & 1.015 & .014 \\
\hline 1816 & 1.006 & 0.867 & 0.870 & 0.925 & 39.00 & 0.965 & 39.48 & 38.46 & 1.015 & 1.014 \\
\hline 1821 & & 0.855 & 84 & & 39.00 & & 40.22 & & & .014 \\
\hline 1826 & 1.006 & 0.855 & 0.864 & 0.857 & 39.00 & 0.965 & 37.30 & 37.87 & 1.014 & 1.014 \\
\hline 1831 & 1.006 & 0.860 & 0.877 & 0.840 & 39.00 & 0.965 & 36.03 & 37.55 & 1.012 & 1.014 \\
\hline 1836 & 007 & 86 & & & 39.00 & & 77 & 37.21 & & .014 \\
\hline 1841 & 1.007 & 0.838 & 0.859 & 0.894 & 40.57 & 0.967 & & & 011 & 1.013 \\
\hline 1846 & .007 & 0.826 & 847 & 0.886 & 38.99 & & 35 & 36.47 & 12 & 1.013 \\
\hline 1851 & 1.008 & 0.829 & 00 & & 40.19 & & 8 & 05 & 1.016 & .013 \\
\hline 1856 & 1.008 & 0.830 & 856 & 0.8 & 40.63 & & 35 & 35.57 & 1.012 & 1.013 \\
\hline 1861 & 00 & 0.83 & 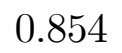 & & 40.48 & & 36.30 & & & 1.013 \\
\hline 1866 & 1.009 & 0.826 & 0.8 & 09 & 39.93 & 0.9 & & & & 1.012 \\
\hline 1871 & 1.01 & 0.83 & 0.874 & 913 & 40.01 & & o & 67 & - & 1.012 \\
\hline 1876 & 1.010 & .83 & 0.88 & & 40.29 & & 36.30 & & 1.015 & 1.012 \\
\hline 1881 & 1010 & 084 & & & & & & & 1.012 & 1.011 \\
\hline 188 & 1 & 0.84 & 89 & 1 & 41. & & 80 & 38 & 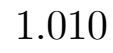 & 1.011 \\
\hline 18 & 1 & 0.83 & 90 & 42 & 41 & 0. & 31 & 76 & $a$ & 1.010 \\
\hline & & & & & 42 & & & & & 1.009 \\
\hline 190 & 010 & .84 & 913 & & 43.43 & 0. & 28.50 & 27.32 & 1.010 & 1.009 \\
\hline 1906 & & & & & & & & & 10 & 008 \\
\hline & 011 & 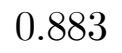 & & & 45.37 & & & & & 1.008 \\
\hline $1 ?$ & 1.011 & 0.9 & 0 & 0 & 38 & & 20.90 & 23.47 & 34 & 1.007 \\
\hline & & & & & & & & & & 1.007 \\
\hline $1 !$ & 011 & 0.92 & & & 47.85 & & 17 & 2 & 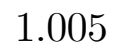 & 1.007 \\
\hline 1931 & 1.012 & 0.935 & 0 & 70 & & & & & 04 & 1.006 \\
\hline & & & & & & & & & & 1.006 \\
\hline & 13 & 0 & & & 4 & & 1 & 9 & 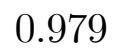 & 1.006 \\
\hline & & 0 & & & 50 & & & & & 1.006 \\
\hline & & & & & & & & & & 1006 \\
\hline 1956 & 1.015 & 0.976 & 0.996 & 3 & 53.17 & & 15.70 & 87 & 05 & 1.006 \\
\hline & & & & & & & & & & 1.005 \\
\hline & & & & & 5 & & & & & 1.005 \\
\hline 1 ? & 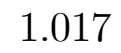 & 0 & & & 54.26 & & & & & 1.005 \\
\hline & & & & & & & & & & \\
\hline & & & & & & & & & & 1.004 \\
\hline 198 & 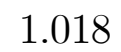 & & & & 56.46 & & & & & 1.003 \\
\hline 1991 & 1.018 & 0.993 & 0.999 & 0.996 & 57.39 & 0.979 & 13.20 & 12.15 & 1.004 & 1.003 \\
\hline
\end{tabular}




\section{A.5 Perfect Foresight Transition Paths to BGP}

In this section, we discuss an alternative to the calculation given in section 6 . There, the simulated data were calculated assuming that the agents believed that their current circumstances, in terms of child costs, productivity growth rates and survival probabilities, would prevail indefinitely into the future when making their decisions - i.e., the calculations are BGP to BGP. The weakness of this is that it assumes that agents act as if circumstances will not change in the future, even though they actually will. At the other extreme, one could assume that agents in a give period $t$, anticipate exactly all future changes that will occur - i.e., there is perfect foresight with respect to future values of $\gamma, \pi$ and $\theta_{s}$. Here we give the calculations for the model under this alternative assumption. We find that this makes very little difference in the end.

From the Planner's problem in Section 4.1, the FOC for $N_{t+1}$ is:

$$
\theta_{s, t} N_{t}^{\eta+\sigma-1} C_{t}^{-\sigma}=\beta\left[\frac{(\eta+\sigma-1)}{(1-\sigma)} N_{t+1}^{\eta+\sigma-2} C_{t+1}^{1-\sigma}+\left[w_{t+1}+\theta_{s, t+1} \pi_{t+1}\right] N_{t+1}^{\eta+\sigma-1} C_{t+1}^{-\sigma}\right]
$$

The other equation determining the solution is:

$$
C_{t}=\left[w_{t}+\theta_{s, t} \pi_{t}\right] N_{t}-\theta_{s, t} N_{t+1}
$$

After some algebra, these two equations can be rewritten as:

$$
\begin{aligned}
& \gamma_{N t}^{1-\eta}\left[\frac{\left[\frac{w_{t+1}}{\theta_{s, t+1}}+\pi_{t+1}\right]-\gamma_{N t+1}}{\left[\frac{w_{t}}{\theta_{s, t}}+\pi_{t}\right]-\gamma_{N t}}\right]^{\sigma} \\
& =\beta\left[\frac{\theta_{s, t+1}}{\theta_{s, t}}\right]^{1-\sigma}\left[\frac{(\eta+\sigma-1)}{(1-\sigma)}\left[\left[\frac{w_{t+1}}{\theta_{s, t+1}}+\pi_{t+1}\right]-\gamma_{N t+1}\right]+\left[\frac{w_{t+1}}{\theta_{s, t+1}}+\pi_{t+1}\right]\right] \\
& \frac{C_{t}}{N_{t}} \frac{1}{\theta_{s, t}}=\left[\frac{w_{t}}{\theta_{s, t}}+\pi_{t}\right]-\gamma_{N t} .
\end{aligned}
$$

The first of these is a first order difference equation in $\gamma_{N}$. It has time varying coefficients however.

If $\left(\theta_{s, t}, w_{t}, \pi_{t}\right)$ converge in the sense that $\frac{w_{t}}{\theta_{s, t}} \rightarrow \frac{w}{\theta_{s}}, \frac{\theta_{s, t+1}}{\theta_{s, t}} \rightarrow \gamma, \pi_{t} \rightarrow \pi$, it can be shown that the solution to the model converges to the BGP determined by $\frac{w}{\theta_{s}}, \gamma$, and $\pi$. Further, assuming that $\frac{w_{t}}{\theta_{s, t}}=\frac{w}{\theta_{s}}, \frac{\theta_{s, t+1}}{\theta_{s, t}}=\gamma$, and $\pi_{t}=\pi$ for all $t \geq t^{*}$ for some $t^{*}$, it can be shown that all of the relevant variables, measured in per capita terms, are constant after date $t^{*}$. Because of this, the model can be solved backwards from $t^{*}$ in this case. Thus, suppose that the sequence of exogenous parameters is given by:

$$
\left(\theta_{s, 0}, w_{0}, \pi_{0}, \ldots, \theta_{s, t^{*}}, w_{t^{*}}, \pi_{t^{*}}, \gamma \theta_{s, t^{*}}, \gamma w_{t^{*}}, \pi_{t^{*}}, \ldots\right) .
$$

Then, the solution to the perfect foresight infinite horizon problem is of the form:

$$
\left(C_{0}, N_{0}, \ldots ., C_{t^{*}}, N_{t^{*}}, C_{t^{*}+1}, N_{t^{*}+1}, \ldots\right)
$$

where:

1) for $t \geq t^{*}+1, N_{t+1}=\gamma_{N} N_{t}$ with $\gamma_{N}$ given by the solution to:

$$
\gamma_{N}^{1-\eta}=\beta \gamma^{1-\sigma}\left[\frac{(\eta+\sigma-1)}{(1-\sigma)}\left[\left[\frac{w_{t^{*}}}{\theta_{s, t^{*}}}+\pi_{t^{*}}\right]-\gamma_{N}\right]+\left[\frac{w_{t^{*}}}{\theta_{s, t^{*}}}+\pi_{t^{*}}\right]\right] \text {; }
$$


2) $\quad \frac{C_{t^{*}}}{N_{t^{*}}} \frac{1}{\theta_{s, t^{*}}}=\frac{C_{t^{*}}^{*}}{N_{t^{*}}} \frac{1}{\theta_{s, t^{*}}}=\left[\frac{w_{t^{*}}}{\theta_{s, t^{*}}}+\pi_{t^{*}}\right]-\gamma_{N}$;

3) for $s \geq 1$,

$$
\frac{C_{t^{*}}^{*}}{N_{t^{*}}} \frac{1}{\theta_{s, t^{*}}}=\left[\frac{w_{t^{*}}}{\theta_{s, t^{*}}}+\pi_{t^{*}}\right]-\gamma_{N} \Leftrightarrow \frac{C_{t^{*}+s}}{N_{t^{*}+s}} \frac{1}{\gamma^{s} \theta_{s, t^{*}}}=\left[\frac{w_{t^{*}}}{\theta_{s, t^{*}}}+\pi_{t^{*}}\right]-\gamma_{N}
$$

4) For $t<t^{*}, \gamma_{N t}$ evolves according to the difference equation:

$$
\begin{aligned}
& \gamma_{N t}^{1-\eta}\left[\frac{\left[\frac{w_{t+1}}{\theta_{s, t+1}}+\pi_{t+1}\right]-\gamma_{N t+1}}{\left[\frac{w_{t}}{\theta_{s, t}}+\pi_{t}\right]-\gamma_{N t}}\right]^{\sigma} \\
& =\beta\left[\frac{\theta_{s, t+1}}{\theta_{s, t}}\right]^{1-\sigma}\left[\frac{(\eta+\sigma-1)}{(1-\sigma)}\left[\left[\frac{w_{t+1}}{\theta_{s, t+1}}+\pi_{t+1}\right]-\gamma_{N t+1}\right]+\left[\frac{w_{t+1}}{\theta_{s, t+1}}+\pi_{t+1}\right]\right] ;
\end{aligned}
$$

5) $\quad$ For $t<t^{*}, \frac{C_{t}}{N_{t}}$ is given by:

$$
\frac{C_{t}}{N_{t}} \frac{1}{\theta_{s, t}}=\left[\frac{w_{t}}{\theta_{s, t}}+\pi_{t}\right]-\gamma_{N t} \text {. }
$$

\section{A.5.1 Numerical Implementation}

We keep the length of a period at $T=20$ years. Suppose from $t^{*}=1990$ on the growth rate in productivity, $\gamma$, infant, child and youth mortality $\left(\pi_{i}, \pi_{i c}, \pi_{c y}\right)$ (and hence, detrended costs of raising surviving children, $\theta_{s}$ ) and adult mortality (longevity), $\pi$, are constant. Then, we can use 1) above to solve for the population growth rate, $\gamma_{N}$, on the balanced growth path using parameter values for 1990. We can then use 4) to solve backward for $\gamma_{N t}, t=1970$ using $\gamma_{N t+1}=\gamma_{N t^{*}} t^{*}=1990$ and so on. To do this, we have to make one additional assumption (similar to the balanced growth assumption), namely that base costs of raising children, $\left(\theta_{i}, \theta_{c}, \theta_{y}\right)$ grow at the same rate as wages every period but are otherwise constant while the cost of raising a surviving child, $\theta_{s}$, may vary additionally because youth mortality varies. As in Sections 5.3 and 6, we assume that base costs are constant fractions of calibrated costs when children survive with certainty. The results from this experiment are almost indistinguishable from the BGP to BGP experiment in Section 6. This is not surprising since changes in mortality and productivity growth are very smooth. That is, knowing that mortality and productivity change slightly in the next few periods induces very similar choices to the setting in which people believe today's parameters will prevail forever. Moreover, the length of a period being 20 years implies large discounts of future utility (children's utility) and hence changes expected in the future do not affect current decisions very much. 
Figure A.3: Perfect Foresight versus Balanced Growth to Balanced Growth, $C B R$

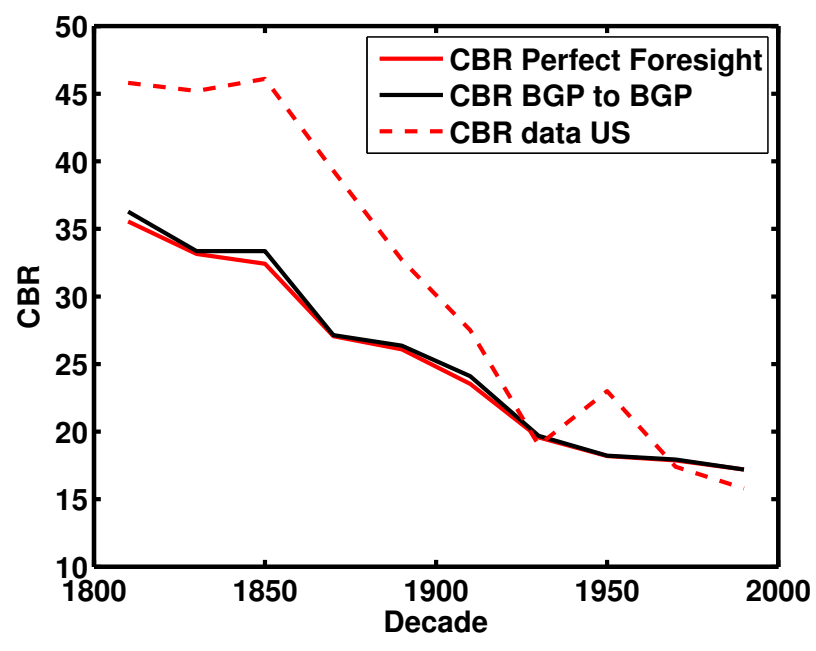

Figure A.4: Perfect Foresight versus Balanced Growth to Balanced Growth, $\gamma_{N}$

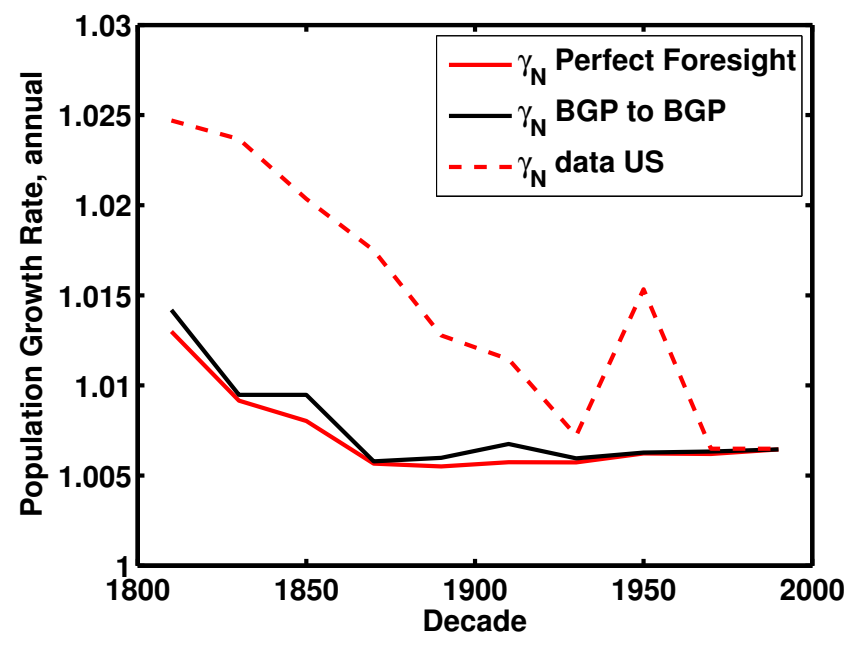

